\title{
Black Hole Growth Is Mainly Linked to Host-galaxy Stellar Mass Rather Than Star Formation Rate
}

\author{
G. Yang ${ }^{1,2}$, C.-T. J. Chen ${ }^{1,2}$, F. Vito ${ }^{1,2}$, W. N. Brandt ${ }^{1,2,3}$, D. M. Alexander ${ }^{4}$, B. Luo ${ }^{5}$, M. Y. Sun ${ }^{6}$, Y. Q. Xue ${ }^{6}$, F. E. Bauer ${ }^{7,8,9}$ \\ A. M. Koekemoer ${ }^{10}$, B. D. Lehmer ${ }^{11}$, T. Liu ${ }^{6}$, D. P. Schneider ${ }^{1,2}$, O. Shemmer ${ }^{12}$, J. R. Trump ${ }^{13}$, C. Vignali ${ }^{14}$, and J.-X. Wang ${ }^{6}$ \\ ${ }^{1}$ Department of Astronomy and Astrophysics, 525 Davey Lab, The Pennsylvania State University, University Park, PA 16802, USA; gxy909@psu.edu \\ ${ }^{2}$ Institute for Gravitation and the Cosmos, The Pennsylvania State University, University Park, PA 16802, USA \\ ${ }^{3}$ Department of Physics, 104 Davey Laboratory, The Pennsylvania State University, University Park, PA 16802, USA \\ ${ }^{4}$ Centre for Extragalactic Astronomy, Department of Physics, Durham University, South Road, Durham DH1 3LE, UK \\ ${ }^{5}$ School of Astronomy \& Space Science, Nanjing University, Nanjing 210093, China \\ ${ }^{6}$ CAS Key Laboratory for Research in Galaxies and Cosmology, Center for Astrophysics, Department of Astronomy, University of Science and Technology of China, \\ Chinese Academy of Sciences, Hefei, Anhui 230026, China \\ ${ }^{7}$ Instituto de Astrofísica and Centro de Astroingeniería, Facultad de Física, Pontificia Universidad Católica de Chile, Casilla 306, Santiago 22, Chile \\ ${ }^{8}$ Millennium Institute of Astrophysics (MAS), Nuncio Monseñor Sótero Sanz 100, Providencia, Santiago, Chile \\ ${ }^{9}$ Space Science Institute, 4750 Walnut Street, Suite 205, Boulder, CO 80301, USA \\ ${ }^{10}$ Space Telescope Science Institute, 3700 San Martin Drive, Baltimore, MD 21218, USA \\ ${ }^{11}$ Department of Physics, University of Arkansas, 226 Physics Building, 835 West Dickinson Street, Fayetteville, AR 72701, USA \\ ${ }^{12}$ Department of Physics, University of North Texas, Denton, TX 76203, USA \\ ${ }^{13}$ Department of Physics, 2152 Hillside Road, U-3046, University of Connecticut, Storrs, CT 06269, USA \\ ${ }^{14}$ Universita di Bologná, Via Ranzani 1, Bologna, Italy \\ Received 2016 December 5; revised 2017 April 12; accepted 2017 May 25; published 2017 June 15
}

\begin{abstract}
We investigate the dependence of black hole accretion rate (BHAR) on host-galaxy star formation rate (SFR) and stellar mass $\left(M_{*}\right)$ in the CANDELS/GOODS-South field in the redshift range of $0.5 \leqslant z<2.0$. Our sample consists of $\approx 18,000$ galaxies, allowing us to probe galaxies with $0.1 M_{\odot} \mathrm{yr}^{-1} \lesssim \mathrm{SFR} \lesssim 100 M_{\odot} \mathrm{yr}^{-1}$ and $/ \mathrm{or}$ $10^{8} M_{\odot} \lesssim M_{*} \lesssim 10^{11} M_{\odot}$. We use sample-mean BHAR to approximate long-term average BHAR. Our samplemean BHARs are derived from the Chandra Deep Field-South 7 Ms observations, while the SFRs and $M_{*}$ have been estimated by the CANDELS team through spectral energy distribution fitting. The average BHAR is correlated positively with both SFR and $M_{*}$, and the BHAR-SFR and BHAR- $M_{*}$ relations can both be described acceptably by linear models with a slope of unity. However, BHAR appears to be correlated more strongly with $M_{*}$ than SFR. This result indicates that $M_{*}$ is the primary host-galaxy property related to supermassive black hole (SMBH) growth, and the apparent BHAR-SFR relation is largely a secondary effect due to the star-forming main sequence. Among our sources, massive galaxies $\left(M_{*} \gtrsim 10^{10} M_{\odot}\right)$ have significantly higher BHAR/SFR ratios than less massive galaxies, indicating that the former have higher SMBH fueling efficiency and/or higher SMBH occupation fraction than the latter. Our results can naturally explain the observed proportionality between $M_{\mathrm{BH}}$ and $M_{*}$ for local giant ellipticals and suggest that their $M_{\mathrm{BH}} / M_{*}$ is higher than that of local star-forming galaxies. Among local star-forming galaxies, massive systems might have higher $M_{\mathrm{BH}} / M_{*}$ compared to dwarfs.
\end{abstract}

Key words: galaxies: active - galaxies: evolution - galaxies: nuclei - quasars: supermassive black holes X-rays: galaxies

\section{Introduction}

The origin of the likely coevolution between supermassive black holes (SMBHs) and their host galaxies remains a fundamental question (e.g., Hopkins et al. 2008; Marulli et al. 2008; Fabian 2012; Kormendy \& Ho 2013). Observations reveal a linear correlation between star formation rate (SFR) and sample-averaged black hole accretion rate $(\langle$ BHAR $\rangle$ ) for star-forming galaxies (e.g., Chen et al. 2013, hereafter C13). Also, X-ray-selected active galactic nuclei (AGNs) preferentially reside in star-forming rather than quiescent galaxies for samples with matched stellar mass ( $M_{*}$; e.g., Rosario et al. 2013), and optically selected luminous quasars tend to be hosted by strongly star-forming systems (e.g., Harris et al. 2016; Netzer et al. 2016). However, the sample-averaged SFR $(\langle\mathrm{SFR}\rangle)$ of the host galaxies of $\mathrm{X}$-ray AGNs does not show a significant dependence on the BHAR in the regimes of low and moderate AGN luminosity, while the potential existence of a positive SFR-BHAR relation at high luminosities is still debatable (e.g., Harrison et al. 2012; Rosario et al. 2012; Barger et al. 2015; Stanley et al. 2015).

To reconcile the apparent discrepancy, Hickox et al. (2014, hereafter H14) proposed a model in which the long-term ( $\sim 100 \mathrm{Myr})$ average BHAR traces SFR linearly, but AGN variability hides the BHAR-SFR relation for individual X-ray AGNs (also see Rosario et al. 2013); SFRs are stable over timescales $\gtrsim 100 \mathrm{Myr}$, while AGNs are variable over much shorter timescales. This simple scenario reasonably explains observations, including both the linear $\langle$ BHAR $\rangle-$ SFR relation for star-forming galaxies and the generally flat $\langle\mathrm{SFR}\rangle-\mathrm{BHAR}$ relation for X-ray-selected AGNs.

The H14 model requires strong AGN variability (by a factor of $\gg 10$ ) on timescales of $\lesssim 10^{7} \mathrm{yr}$ to be commonplace. Although variability studies on the longest available timescales ( $\lesssim 10 \mathrm{yr}$, rest frame) do not directly reveal the prevalence of such variability (e.g., Shemmer et al. 2014; Yang et al. 2016), its occurrence on timescales of $10^{2}-10^{7} \mathrm{yr}$ is plausible from both observational and theoretical points of view (e.g., Martini \& Schneider 2003; Novak et al. 2011). In fact, some 
observational evidence suggests that the typical AGN-phase timescale is $\sim 10^{5}$ year, as expected from the chaotic-accretion scenario (e.g., King \& Nixon 2015; Schawinski et al. 2015). Due to the potential existence of such strong variability, the BHAR derived from direct X-ray observations of individual AGNs might not be a reliable indicator of long-term average $\mathrm{SMBH}$ growth rate. On the other hand, $\langle\mathrm{BHAR}\rangle$, the average BHAR over a sample of galaxies, serves as a proxy for typical long-term average BHAR of the sample (e.g., C13 and H14). Therefore, $\langle\mathrm{BHAR}\rangle$ provides a useful tool to study SMBHgalaxy coevolution.

Another major motivation of the $\mathrm{H} 14$ model is that, in the local elliptical galaxies, the mass of SMBHs $\left(M_{\mathrm{BH}}\right)$ is roughly proportional to the bulge $M_{*}$ (equivalent to host-galaxy $M_{*}$ for ellipticals; see Kormendy \& Ho 2013, for a review). If the long-term average BHAR is proportional to SFR for all galaxies, then a natural consequence is that $M_{\mathrm{BH}}$ correlates with $M_{*}$ linearly, as long as the accreted mass dominates over the mass of SMBH seeds (e.g., Volonteri 2010). However, hints have been found of spiral and dwarf galaxies hosting undermassive SMBHs relative to the $M_{\mathrm{BH}}-M_{*}$ relation derived from ellipticals, although large uncertainties exist (e.g., Greene et al. 2010; Miller et al. 2015; Reines \& Volonteri 2015; Trump et al. 2015; Greene et al. 2016). This behavior is not expected from the H14 model, which assumes that SFR is the only factor determining long-term average BHAR. Also, simulations indicate that the apparent discrepancy between the $\langle$ BHAR $\rangle-$ SFR and the $\langle$ SFR $\rangle$-BHAR relations can be produced by the effect of binning on the intrinsic bivariate relationship between BHAR and SFR (e.g., Volonteri et al. 2015), regardless of whether the intrinsic shape of this distribution is produced by an intrinsic long-term BHAR-SFR relation as proposed by $\mathrm{H} 14$.

Observations show that the fraction of AGNs above a given luminosity threshold rises steeply toward massive galaxies (e.g., Xue et al. 2010; Aird et al. 2012; Bongiorno et al. 2012; Mullaney et al. 2012b). Furthermore, for $M_{*}$-matched samples, the fraction of galaxies hosting AGNs appears to have no dependence on host-galaxy colors (e.g., Silverman et al. 2009; Pierce et al. 2010; Xue et al. 2010). However, apparent galaxy colors might be a poor indicator of SFR, as high-SFR galaxies might appear red owing to significant dust obscuration (e.g., Whitaker et al. 2012; Rosario et al. 2013; and references therein). Therefore, it is still not clear whether $M_{*}$ or SFR is the dominant factor correlated with black hole accretion.

With the advent of deep ultraviolet (UV) to infrared (IR) observations from surveys such as CANDELS (Grogin et al. 2011; Koekemoer et al. 2011), it has become possible to estimate reliably $M_{*}$ and SFR for the majority population of galaxies with acceptable uncertainties $(\lesssim 0.1$ and 0.2 dex for $M_{*}$ and SFR, respectively; see Section 2.2). The uncertainties on $M_{*}$ and SFR are small compared to the parameter ranges probed (both $\approx 3$ orders of magnitude) and thus are acceptable for our analyses. The $7 \mathrm{Ms}$ Chandra Deep Field-South (CDF$\mathrm{S}$, covering the whole CANDELS/GOODS-South region; see Luo et al. 2017, hereafter L17) X-ray survey has achieved unprecedented sensitivities, allowing the derivation of accurate $\langle\mathrm{BHAR}\rangle$ values for the galaxies in CANDELS/GOODS-South. In this paper, we evaluate the dependence of $\langle\mathrm{BHAR}\rangle$ on both SFR and $M_{*}$ for galaxies in CANDELS/GOODS-South. Also,
Table 1

Numbers of Sources in Different Samples

\begin{tabular}{lcccc}
\hline \hline $\begin{array}{l}\text { Sample } \\
(1)\end{array}$ & $\begin{array}{c}\text { Low- } z \\
(2)\end{array}$ & $\begin{array}{c}\text { High- } z \\
(3)\end{array}$ & $\begin{array}{c}\text { Total } \\
(4)\end{array}$ & $\begin{array}{c}\text { Figure(s) } \\
(5)\end{array}$ \\
\hline All & 10057 & 8164 & 18221 & 1 and 2 \\
$0.1 \leqslant \mathrm{SFR}<100 M_{\odot} \mathrm{yr}^{-1}(\mathrm{~A})$ & 6384 & 7541 & 13925 & 4 \\
$10^{8} \leqslant M_{*}<10^{11} M_{\odot}(\mathrm{B})$ & 6445 & 6669 & 13114 & 5 and 8 \\
$(\mathrm{~A}) \wedge(\mathrm{B})$ & 5224 & 6236 & 11460 & 6
\end{tabular}

Note. Column (1): sample definition. Columns (2) and (3): number of sources with $0.5 \leqslant z<1.3$ and $1.3 \leqslant z<2.0$, respectively. Column (4): total number of sources in both redshift ranges. Column (5): relevant figure(s).

we study the efficiency of SMBH growth compared to SFR for galaxies of different $M_{*}$.

The paper is structured as follows. We describe the sample selection and measurements of SFR, $M_{*}$, and BHAR in Section 2. In Section 3, we present the results of our analyses. We discuss scientific implications of our results in Section 4. We summarize our results in Section 5.

Throughout this paper, we assume a cosmology with $H_{0}=70 \mathrm{~km} \mathrm{~s}^{-1} \mathrm{Mpc}^{-1}, \Omega_{M}=0.3$, and $\Omega_{\Lambda}=0.7$ and a Chabrier initial mass function (IMF; Chabrier 2003). Quoted uncertainties are at the $1 \sigma(68 \%)$ confidence level, unless otherwise stated. SFR and BHAR are in units of $M_{\odot} \mathrm{yr}^{-1}$, and $M_{*}$ is in units of $M_{\odot}$, unless otherwise stated.

\section{Data Analyses}

\subsection{Sample Selection}

We first select all galaxies with $0.5 \leqslant z<2.0$ and F160W $<28$ in the CANDELS/GOODS-South catalog (Guo et al. 2013; Santini et al. 2015, hereafter S15). ${ }^{15}$ We do not include sources beyond $z=2$, because $M_{*}$ and SFR values estimated from spectral energy distribution (SED) fitting (Section 2.2) suffer from potential biases in that redshift regime (e.g., Wuyts et al. 2011; S15). The SEDs of broad-line AGNs often have significant accretion disk emission besides their starlight. Their SFR and $M_{*}$ measurements from SED fitting have potential large uncertainties (e.g., Bongiorno et al. 2012; Sun et al. 2015). Thus, we exclude the 19 broadline AGNs reported in the literature (e.g., Mignoli et al. 2005; Ravikumar et al. 2007; Silverman et al. 2010) and discuss the effects of their exclusion in Section 3.5.1. Applying these criteria, we select 18,221 sources (see Table 1), of which 1305 have secure spectroscopic redshifts, and the rest have highquality photometric redshifts based on up to 17 bands from the UV to IR (S15). Compared to spectroscopic redshifts when available, the photometric redshifts have median uncertainty $\left|z_{\text {phot }}-z_{\text {spec }}\right| /\left(1+z_{\text {spec }}\right) \approx 2 \%$ with an outlier (uncertainty $>15 \%$ ) fraction of $3 \%$.

\subsection{Stellar Mass and Star Formation Rate}

We collect the $M_{*}$ and SFR values for our sources from S15, who presented SED-fitting results of several independent teams. We adopt the median values of $M_{*}$ and SFR from the five available teams (i.e., labeled as $2 \mathrm{a}_{\tau}, 6 \mathrm{a}_{\tau}, 11 \mathrm{a}_{\tau}, 13 \mathrm{a}_{\tau}$, and $14 \mathrm{a}$ in S15). All five teams employed stellar templates from

\footnotetext{
$15 \mathrm{~F} 160 \mathrm{~W}=28$ is approximately the $5 \sigma$ limiting magnitude of the CANDELS/GOODS-South catalog (Guo et al. 2013).
} 


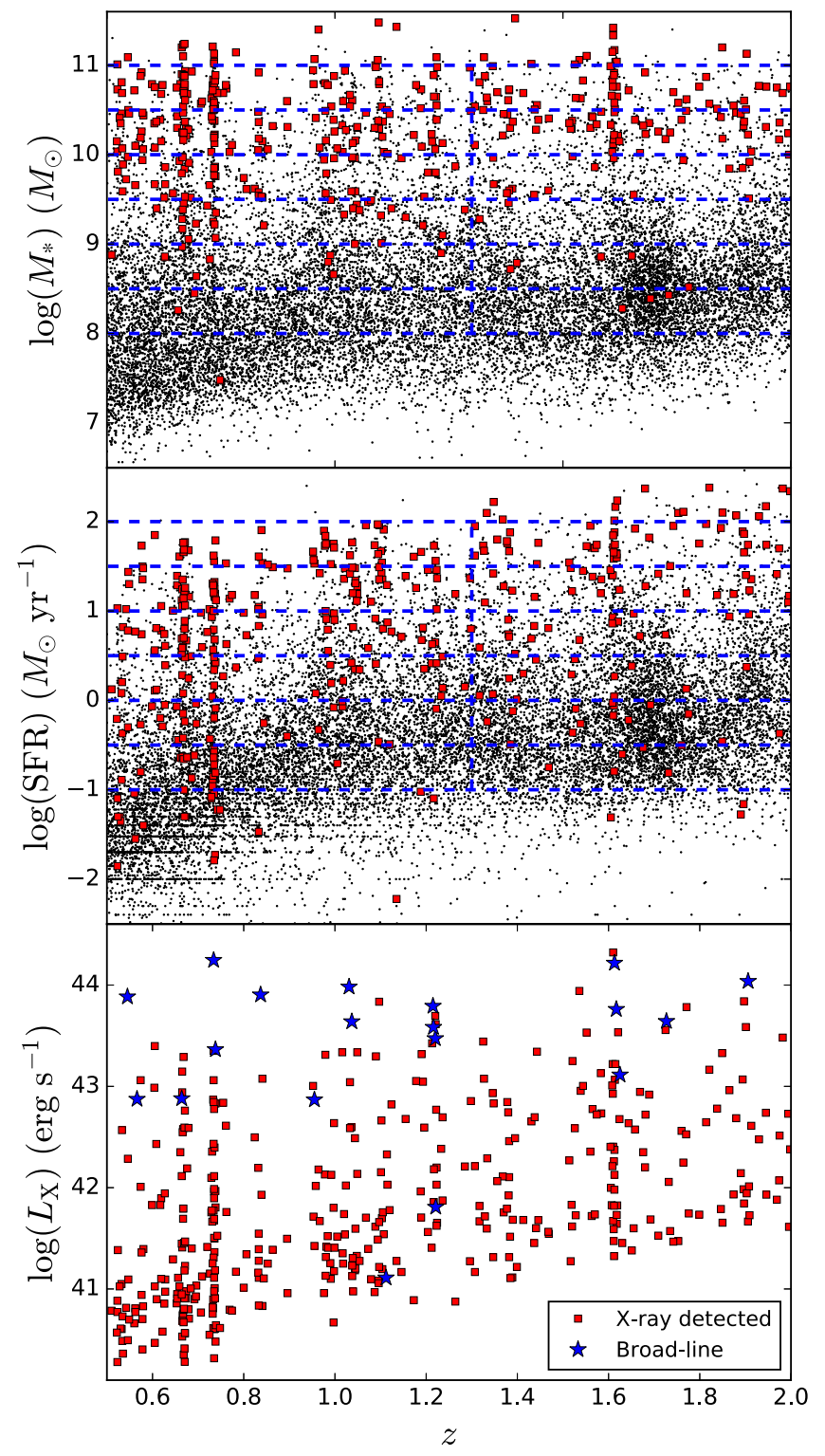

Figure 1. $M_{*}$ (top) and SFR (middle) vs. redshift for all our sources. The red squares indicate X-ray detected sources. The blue dashed lines indicate our binning grids in Figures 4, 5, and 8. The bottom panel presents absorptioncorrected $L_{X}$ (see Section 2.3) as a function of redshift for X-ray detected sources. The blue stars indicate broad-line AGNs that are excluded from our sample. The overdensities at $z \approx 0.7$ and 1.6 are likely due to cosmic variance (e.g., Silverman et al. 2010; Finoguenov et al. 2015; L17).

Bruzual \& Charlot (2003) and a Chabrier IMF when performing the SED fitting. Teams $2 \mathrm{a}_{\tau}, 6 \mathrm{a}_{\tau}, 11 \mathrm{a}_{\tau}$, and $13 \mathrm{a}_{\tau}$ assumed an exponentially declining star formation history ( $\mathrm{SFH}$ ); 14a assumed a more flexible SFH (see S15). Teams $2 \mathrm{a}_{\tau}, 6 \mathrm{a}_{\tau}, 11 \mathrm{a}_{\tau}$, and $14 \mathrm{a}$ adopted a Calzetti extinction law (Calzetti et al. 2000); $13 \mathrm{a}_{\tau}$ adopted a combination of Calzetti and SMC extinction laws. The $M_{*}$ and SFR values estimated by the five teams agree well; their typical deviations from the adopted medians are $\lesssim 0.1$ dex and $\lesssim 0.2$ dex, respectively. These values largely represent uncertainties arising from SED fitting, and additional systematic errors (from, e.g., IMF and SFH assumptions) likely exist. However, such systematic uncertainties should not affect our conclusions qualitatively (see Section 3). Figure 1 shows $M_{*}$ and SFR as functions of redshift, and Figure 2 shows the $M_{*}-$ SFR

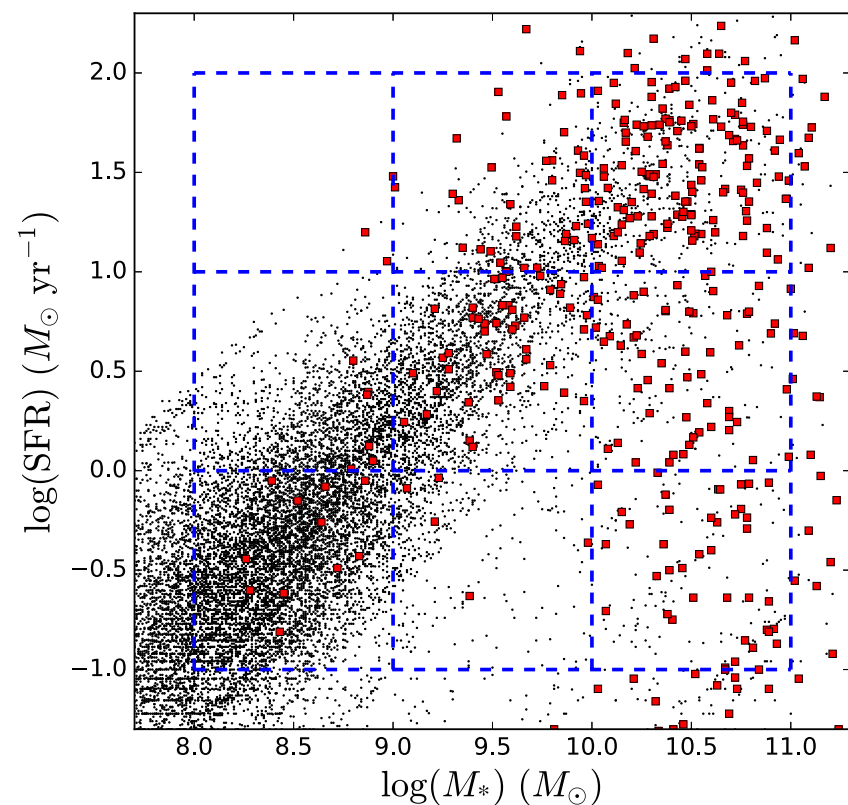

Figure 2. SFR vs. $M_{*}$ for all our sources. The red squares indicate X-ray detected sources. The blue dashed lines indicate our binning grids in Figure 6 .

plane for all our sources. From Figures 1 (top panel) and 2, X-ray detected sources ${ }^{16}$ are preferentially found among massive galaxies (also see, e.g., Xue et al. 2010; Aird et al. 2012). Our sample is roughly complete for galaxies with $M_{*} \gtrsim 10^{8} M_{\odot}$ and $\mathrm{SFR} \gtrsim 0.1 M_{\odot} \mathrm{yr}^{-1}$ (i.e., the $M_{*}$ and SFR regimes mostly probed by our analyses; see Section 3). A more detailed discussion of completeness is presented in Section 3.5.2.

The rest-frame UV to near-IR SEDs $(\approx 0.2-4 \mu \mathrm{m}$, similar to the wavelength range used by S15 to derive $M_{*}$ and SFR) of $\mathrm{X}$-ray-selected AGNs in the CDF-S are usually dominated by stellar light (see Figure 9 of Luo et al. 2010; also see, e.g., Xue et al. 2010; Brandt \& Alexander 2015). In addition, we have excluded broad-line AGNs (Section 2.1), because their $M_{*}$ and SFR measurements from SED fitting could be overestimated (e.g., Ciesla et al. 2015). Therefore, the CANDELS $M_{*}$ and SFR should not have significant biases due to AGN activity. Also, we confirm that AGNs do not have biased SED-based SFRs in comparison with SFRs based on far-IR (FIR) photometry (see below).

As demonstrated by $\mathrm{S} 15$, the CANDELS $M_{*}$ values generally have high quality. To evaluate the accuracy of CANDELS SED-based SFRs, which ultimately come from dust-corrected UV luminosities, we compare them with those obtained from FIR photometry. We match our sources with the Herschel/PACS catalog of the PACS Evolutionary Probe (PEP; e.g., Lutz et al. 2011; Magnelli et al. 2013) survey using a $2^{\prime \prime}$ matching radius. ${ }^{17}$ We follow the method of $\mathrm{C} 13$ to derive FIR-based SFRs for the matched sources. Briefly, we convert the PACS band flux to total IR luminosity according to the starforming galaxy spectral template from Kirkpatrick et al. (2012), ${ }^{18}$ and then we scale the IR luminosity to SFR following the relation from Kennicutt (1998) (modified for our Chabrier

\footnotetext{
${ }^{16}$ These are sources presented in the $7 \mathrm{Ms} \mathrm{CDF}-\mathrm{S}$ main catalog (Section 2.3), formally defined with "binomial no-source probability" $P_{\mathrm{B}}<0.007$ (see L17). ${ }_{17}$ We use the $24 \mu \mathrm{m}$ prior PEP catalog owing to its good positional accuracy.

18 Kirkpatrick et al. (2012) present two templates at $z \sim 1$ and $z \sim 2$, respectively. Here, we use the $z \sim 1$ template following $\mathrm{C} 13$, although the $z \sim 2$ template leads to almost the same results.
} 


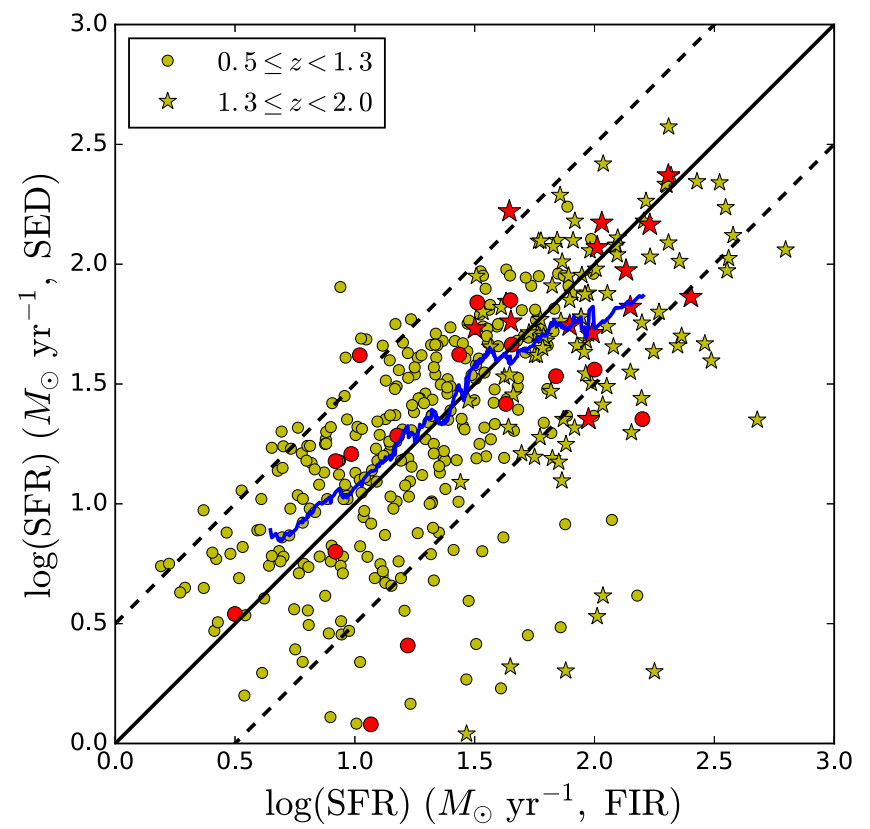

Figure 3. Comparison between SFRs derived from SED fitting and those derived from FIR photometry. Circles and stars indicate sources in the redshift ranges of $0.5 \leqslant z<1.3$ and $1.3 \leqslant z<2.0$, respectively. Their FIR-based SFRs are derived from 100 and $160 \mu \mathrm{m}$ observations with Herschel/PACS, respectively (see Section 2.2). Red symbols indicate AGNs selected by their large X-ray luminosities $\left(L_{\mathrm{X}}>3 \times 10^{42} \mathrm{erg} \mathrm{s}^{-1}\right.$; see, e.g., L17). The black solid line indicates a 1:1 relation between two SFR measurements; the dashed lines indicate 0.5 dex offsets. Our adopted SFRs (from SED fitting) generally agree with those derived from FIR photometry. The blue solid curve indicates running median SFR offsets from bins of 50 sources. For X-ray-luminous AGNs, the SED-based SFRs do not have significant systematic differences relative to FIR-based SFRs.

IMF; see C13). We use the PACS $100 \mu \mathrm{m}$ band for sources at $0.5 \leqslant z<1.3$ and the $160 \mu \mathrm{m}$ band at $1.3 \leqslant z<2.0$, requiring photometric $\mathrm{S} / \mathrm{N}>5$. Using different bands for different redshift ranges is to sample the SED peak $(\approx 50-80 \mu \mathrm{m}$, rest frame; e.g., Kirkpatrick et al. 2012) of cold dust emission, a good proxy for star formation. The FIR-based SFR estimation assumes that dust absorbs most UV photons and reemits IR radiation. It is thus robust for galaxies with relatively high SFR $\left(\gtrsim 1 M_{\odot} \mathrm{yr}^{-1}\right.$ ) where dust is often abundant (e.g., Calzetti et al. 2010; Kennicutt \& Evans 2012), and this is indeed our case, since low-SFR galaxies within our redshift range $(0.5 \leqslant z<2.0)$ are usually not detected by Herschel.

Figure 3 compares CANDELS SED-based SFRs with FIRbased SFRs for Herschel-detected galaxies that probe the SFR range of $\approx 10^{0.5}-10^{2.5} M_{\odot} \mathrm{yr}^{-1}$. CANDELS SFRs generally agree well with FIR-based SFRs without significant systematic bias: the median offset between them is 0.01 dex. The median offset depends on SFR (the blue solid curve in Figure 3). The offset is small $(\lesssim 0.2$ dex $)$ at low and intermediate SFR, but becomes $\approx 0.3 \mathrm{dex}$ at SFR $\sim 100 M_{\odot} \mathrm{yr}^{-1}$. The median offsets for the low $z$ and high- $z$ bins are 0.07 and 0.16 dex, respectively. A similar systematic offset in the high-SFR (also high-z) regime is also found by Wuyts et al. (2011), possibly because dust correction cannot fully recover the intrinsic UV luminosity when the obscuration is very strong. Nevertheless, the systematic errors are smaller than our bin width of SFR (i.e., 0.5 and 1 dex; see Section 3) and thus should not affect our results significantly.

For most sources $(80 \%)$, the SFRs derived by the two methods agree within 0.5 dex (the dashed lines in Figure 3).
The outliers (20\%) tend to have FIR-based SFRs higher than SED-based SFRs, possibly because the FIR sample is fluxlimited and FIR-luminous outliers are more likely to be detected by Herschel (see also, e.g., Azadi et al. 2015). There are $6 \%$ extreme outliers with SFR offsets larger than 1 dex. For these sources, the SFR measurements from different SEDfitting teams are often inconsistent; their typical deviations from the adopted medians are $\approx 0.3-1$ dex, significantly larger than those for the whole sample $(\lesssim 0.2 \mathrm{dex})$. The likely failure of SED fitting might be caused by inappropriate model assumptions in SED fitting, e.g., SFH and extinction law. False matches between the CANDELS and FIR catalogs might also be responsible for some of the extreme outliers. Nevertheless, none of these extreme outliers have high X-ray luminosities $\left(>3 \times 10^{42} \mathrm{erg} \mathrm{s}^{-1}\right)$, and their large SFR uncertainties are unlikely to affect our results qualitatively. Notably, for AGN-dominated X-ray sources with $L_{\mathrm{X}}$ $>3 \times 10^{42} \mathrm{erg} \mathrm{s}^{-1}$ (see L17; red symbols in Figure 3), the CANDELS SFRs do not show a significant systematic bias relative to SFRs derived from FIR photometry: the median offset is $0.01 \mathrm{dex}$, similar to the value for all Herschel-detected galaxies above. Therefore, the SED-based SFRs are reliable for galaxies with SFR $\gtrsim 10^{0.5} M_{\odot} \mathrm{yr}^{-1}$. In the lower-SFR regimes, corrections for dust extinction are generally low or moderate, and SED-based SFRs are generally reliable (e.g., Wuyts et al. 2011; Kennicutt \& Evans 2012).

\subsection{Black Hole Accretion Rate}

We use X-ray observations to derive $\langle$ BHAR $\rangle$ for our sources. We match our 18,221 galaxies with the 7 Ms main source catalog for the CDF-S (L17) using a 0."5 matching radius, and $395 \mathrm{X}$-ray sources are matched. ${ }^{19} \mathrm{~A}$ total of 259 of the 395 X-ray detected sources have spectroscopic redshifts. The host-galaxy properties $\left(z, M_{*}\right.$, and SFR) of these X-ray detected sources are shown in Figures 1 and 2.

We fit their unbinned X-ray spectra (observed-frame 0.5-7 $\mathrm{keV}$ ) with the Cash statistic (Cash 1979). We perform the fitting with a standard absorbed power-law model (i.e., wabs $\times$ zwabs $\times$ powerlaw in XSPEC; see Arnaud 1996, for a description of XSPEC) to recover their absorption-corrected X-ray luminosities $\left(L_{\mathrm{X}}\right.$, rest-frame $2-10 \mathrm{keV}$; e.g., Yang et al. 2016). The wabs component accounts for Galactic absorption with absorption column density $\left(N_{\mathrm{H}}\right)$ set to $8.8 \times 10^{19} \mathrm{~cm}^{-2}$ (Stark et al. 1992). The zwabs component models intrinsic absorption (i.e., wabs at redshift $z$ ). The normalization of powerlaw, intrinsic photon index, and intrinsic $N_{\mathrm{H}}$ are free parameters in the fitting. The allowed ranges of photon index and $N_{\mathrm{H}}$ are set to 1.4-2.2 and $10^{19}-10^{24}$ $\mathrm{cm}^{-2}$, respectively. We then obtain the $L_{\mathrm{X}}$ with XSPEC from the best-fit model parameters. The best-fit $L_{X}$ as a function of redshift is shown in Figure 1 (bottom). Thanks to the great sensitivity of the $7 \mathrm{Ms}$ CDF-S survey, sources with low X-ray luminosities $\left(\lesssim 3 \times 10^{42} \mathrm{erg} \mathrm{s}^{-1}\right)$ can be detected up to $z=2$.

\footnotetext{
${ }^{19}$ We use the positions of the L17 optical/near-infrared counterparts rather than the X-ray positions, because the former are more accurate. In the CANDELS/GOODS-South field, there are 704 out of the 1008 X-ray sources in the L17 catalog, and most of them $(674 / 704=96 \%)$ have CANDELS counterparts. The remaining 30 sources without counterparts might be faint (F160W band) sources not detected by CANDELS, nearby off-nuclear sources (e.g., ultraluminous X-ray sources), or false X-ray detections (L17). A total of 395 of the 674 X-ray sources with CANDELS counterparts are galaxies in our redshift range $(0.5 \leqslant z<2.0)$ without broad lines reported in their spectra.
} 
$\mathrm{X}$-ray emission at this low level might not be dominated by AGNs, but could also originate from stellar processes.

Our absorbed power-law model is appropriate for moderately obscured or unobscured AGNs; it might result in unreliable $L_{\mathrm{X}}$ for Compton-thick AGNs (CTK AGNs; i.e., $N_{\mathrm{H}} \gtrsim 10^{24} \mathrm{~cm}^{-2}$ ). However, it is generally challenging to identify bona fide CTK AGNs among the X-ray detected sources in deep fields such as CDF-S, due to the limited numbers of counts available, and there is no strong evidence suggesting that CTK AGNs are the dominant population (e.g., Alexander et al. 2013; Brightman et al. 2014; Section 3.3 of Brandt \& Alexander 2015). We have tested a basic CTK model, wabs $\times$ zwabs $\times$ pexmon, on our $\mathrm{X}$ ray detected sources (for pexmon, see Magdziarz \& Zdziarski 1995; Nandra et al. 2007; for model parameter settings see Appendix B of Yang et al. 2016). Only 4\% of sources show statistically significant improvements in fitting compared to our adopted wabs $\times$ zwabs $\times$ powerlaw model, where we use the Akaike information criterion to infer fitting improvement (see Yang et al. 2016, for details). Thus, CTK sources are not likely to be the dominant population in our sample. Depending on model assumptions (e.g., obscuration geometry and viewing angle), the CTK models available often have large uncertainties in the derived physical parameters, e.g., $L_{\mathrm{X}}$ and $N_{\mathrm{H}}$ (see, e.g., Murphy \& Yaqoob 2009). Therefore, we do not adopt the spectral-fitting results from the CTK models. A discussion on the effects of X-ray undetected CTK sources is presented in Section 3.5.1.

For sources without X-ray detections in each sample in Section 3, we perform a stacking analysis to derive their total $L_{\mathrm{X}}$ following the procedures of Vito et al. (2016). Briefly, we convert the stacked total count rate of the soft band ${ }^{20}$ to total $L_{X}\left(L_{X}\right.$, stack $)$ of the stacked sources assuming their median redshift ${ }^{21}$ and a power-law spectrum with an effective photon index of $\Gamma=1.8$. By setting $\Gamma=1.8$, we assume that the stacked signals are mainly from X-ray emission of X-ray binaries (XRBs) and/or AGNs with low obscuration of $N_{\mathrm{H}} \lesssim 10^{21.5} \mathrm{~cm}^{-1}$ (see Section 6.1 of Lehmer et al. 2016, for a detailed discussion). Indeed, our stacked X-ray fluxes are similar to those expected from XRBs (see Section 3.1 and 3.2). Even if we adopt a very flat spectral shape of $\Gamma=1$ (i.e., assume that all the stacked X-ray signals are entirely caused by moderately obscured AGNs with $N_{\mathrm{H}} \sim 10^{22.5} \mathrm{~cm}^{-2}$ ), the resulting $L_{\mathrm{X} \text {, stack }}$ will be only $\sim 0.3$ dex higher, unlikely to have a large impact on our qualitative results (see Section 3). We discuss the potential effects of heavily obscured AGNs that might not be included in our analyses in Section 3.5.1. Following Vito et al. (2016), we exclude sources that are at off-axis angles greater than 7!.8 or close to X-ray detected sources (for the specific criteria see Vito et al. 2016) in the stacking analyses. Those excluded sources represent a real population of galaxies, and thus their contribution to $\langle$ BHAR $\rangle$ should be included in our analyses. We account for the excluded sources $(\leqslant 25 \%$ of the X-ray-undetected sources) in each sample by assuming that their mean $L_{X}$ is the same as that of the stacked sources, and we scale the $L_{\mathrm{X} \text {,stack }}$ by multiplying by $N_{\text {non }} / N_{\text {stack }}$ to obtain the total $L_{\mathrm{X}}$ of all X-rayundetected sources (Equation (1)).

\footnotetext{
${ }^{20}$ Observed-frame $0.5-2 \mathrm{keV}$. The soft band has larger collecting area and lower background than the hard band (observed-frame $2-7 \mathrm{keV}$ ), e.g., the expected count rate in the soft band is $\approx 2$ times larger than that in the hard band for a $\Gamma=1.8$ power-law spectrum. We have tested stacking the count rate in the hard band. However, the resulting $\mathrm{S} / \mathrm{N}$ is generally much weaker than that from soft-band stacking, and the hard-band stacked count rates are consistent with zero in many cases.

${ }^{21}$ The median and mean redshifts for our samples (Section 3) are similar, and they only differ by $\lesssim 0.02$.
}

XRBs and other stellar processes in galaxies also contribute to the observed $L_{X}$, and thus we need to subtract their contribution $\left(L_{\mathrm{X}, \mathrm{XRB}}\right)^{22}$ The $L_{\mathrm{X}}, \mathrm{XRB}$ is estimated as $L_{\mathrm{X}, \mathrm{XRB}}$ $=\alpha M_{*}+\beta$ SFR, where $\alpha$ and $\beta$ are coefficients as functions of redshift. We adopted the redshift-dependent $\alpha$ and $\beta$ values from model 269 of Fragos et al. (2013) (corrected to our Chabrier IMF following the prescriptions from Longhetti \& Saracco 2009; Madau \& Dickinson 2014) that is preferred by the observations of Lehmer et al. (2016). ${ }^{23}$ Our adopted $\alpha$ and $\beta$ are also consistent with the values from Aird et al. (2016): the differences are $\approx 0.1$ dex for both $\alpha$ and $\beta$ in $0.5 \leqslant z<2.0$. The mean AGN $L_{\mathrm{X}}$ for each sample is calculated as

$$
\left\langle L_{\mathrm{X}}\right\rangle=\frac{\left(\sum_{\text {detect }} L_{\mathrm{X}}\right)+\frac{N_{\text {non }}}{N_{\text {stack }}} L_{\mathrm{X}, \text { stack }}-\sum_{\text {all }} L_{\mathrm{X}, \mathrm{XRB}}}{N_{\text {detect }}+N_{\text {non }}},
$$

where $N_{\text {detect }}, N_{\text {non }}$, and $N_{\text {stack }}$ are the numbers of X-raydetected, undetected, and stacked sources in the sample, respectively. We do not exclude the 18 radio-loud AGNs identified by Bonzini et al. (2013) in our analyses, although excluding them would have only minor effects on our results. Jet-linked X-ray emission might contribute to their $L_{\mathrm{X}}$, but, at least for the two X-ray brightest radio-loud AGNs, detailed studies do not reveal significant jet-linked X-ray emission (e.g., Iwasawa et al. 2015).

We convert $\left\langle L_{X}\right\rangle$ to mean BHAR as

$$
\begin{aligned}
\langle\mathrm{BHAR}\rangle & =\frac{(1-\epsilon) k_{\mathrm{bol}}\left\langle L_{\mathrm{X}}\right\rangle}{\epsilon c^{2}} \\
& =\frac{3.53\left\langle L_{\mathrm{X}}\right\rangle}{10^{45} \mathrm{erg} \mathrm{s}^{-1}} M_{\odot} \mathrm{yr}^{-1},
\end{aligned}
$$

where we assume a constant bolometric correction factor of $k_{\text {bol }}=22.4$ (the median value for the local AGN sample with $L_{\mathrm{X}} \approx 10^{41}-10^{45} \mathrm{erg} \mathrm{s}^{-1}$ in Vasudevan \& Fabian 2007) and a constant mass-energy conversion efficiency of $\epsilon=0.1$ (e.g., Marconi et al. 2004; Davis \& Laor 2011). We obtain the $1 \sigma$ confidence interval as the range between the 16th and 84th percentiles of the bootstrapped $\langle\mathrm{BHAR}\rangle$ distribution. To obtain the $\langle\mathrm{BHAR}\rangle$ distribution, we randomly resample the sources 1000 times. In this routine procedure of bootstrapping, each random resampling includes the same number of sources as the original sample but allowing repetition of sources. We then calculate $\langle\mathrm{BHAR}\rangle$ with Equations (1) and (2) for each resampling and obtain the $\langle\mathrm{BHAR}\rangle$ distribution.

From Equation (2), we obtain $\langle\mathrm{BHAR}\rangle$ from $\left\langle L_{X}\right\rangle$. This is because X-ray emission is almost a universal tracer of black hole accretion (e.g., Gibson et al. 2008). X-rays are also relatively less affected by obscuration compared to the UV/optical bands and suffer minimal starlight dilution (e.g., Brandt \& Alexander 2015). Nevertheless, there might be uncertainties in the conversion factors $\left(k_{\text {bol }}\right.$ and $\left.\epsilon\right)$ between $\langle$ BHAR $\rangle$ and $\left\langle L_{X}\right\rangle$. The $k_{\text {bol }}=22.4$ and $\epsilon=0.1$ assumptions have been widely adopted in previous studies related to black hole accretion (e.g., Mullaney et al. 2012a and C13). We have also tested applying a luminositydependent $k_{\text {bol }}$ (Hopkins et al. 2007) for the AGN-dominated

\footnotetext{
${ }^{22}$ X-ray emission from XRBs usually dominates over that from other stellar processes. We assume that all non-AGN X-rays are XRB contributions.

${ }^{23}$ We have also tested a simpler $L_{\mathrm{X}}$, XRB model (Ranalli et al. 2003) in which $\alpha$ is zero and $\beta$ is a constant (i.e., not dependent on redshift). Our results below only change slightly using this model.
} 
sources with $L_{X}>3 \times 10^{42} \mathrm{erg} \mathrm{s}^{-1}$ (e.g., L17), and our results do not change qualitatively. Applying the luminosity-dependent $k_{\text {bol }}$ to low-luminosity sources requires careful subtraction of $L_{\mathrm{X}, \mathrm{XRB}}$ for each individual source, but this correction is beyond the scope of our analyses. For simplicity and consistency over all sources, we adopt a constant $k_{\text {bol }}$ in our analyses.

Since we include all X-ray detected and undetected sources (Equation (1)), we are measuring $\langle\mathrm{BHAR}\rangle$ averaged over all galaxies, including systems with both high and low levels of nuclear activity. This is designed to approximate the long-term average BHAR for the entire galaxy sample rather than the instantaneous BHAR for individual AGNs (see Section 1).

\section{Results}

\subsection{BHAR Dependence on SFR}

We bin our sources in six different SFR intervals (bin width $=0.5 \mathrm{dex}$; see Figure 1) for two different redshift ranges $(0.5 \leqslant z<1.3$ and $1.3 \leqslant z<2.0)$ and calculate the $\langle\mathrm{BHAR}\rangle$ for each of the 12 bins, which together include 13,925 sources (Table 1) using Equations (1) and (2). In the following analyses, we discard all bins that have fewer than 100 sources to avoid large statistical fluctuations, unless otherwise stated. ${ }^{24}$ This selection is the main reason why we cannot probe the high-SFR (SFR $>100$ $\left.M_{\odot} \mathrm{yr}^{-1}\right)$ and high- $M_{*}\left(M_{*}>10^{11} M_{\odot}\right)$ regimes (Section 3.2).

Figure 4 displays the results. In general, galaxies with higher SFR have higher $\langle$ BHAR $\rangle$. We perform a least- $\chi^{2}$ fitting for the $\langle$ BHAR $\rangle-S F R$ relation with a linear model ${ }^{25}$ and obtain

$$
\log (\langle\mathrm{BHAR}\rangle)=(0.93 \pm 0.08) \log (\mathrm{SFR})-(3.85 \pm 0.07)
$$

with reduced $\chi^{2}=1.48$, which corresponds to a modelrejection $p$-value of $15 \%$. The slope is consistent with unity. To compare with the H14 model, which assumes that $\langle\mathrm{BHAR}\rangle$ is proportional to SFR $(\langle\mathrm{BHAR}\rangle=\mathrm{SFR} / 3000)$, we fix the slope to 1 and refit the data. This fit results in

$$
\log (\langle\mathrm{BHAR}\rangle)=\log (\langle\mathrm{SFR}\rangle)-(3.89 \pm 0.07)
$$

(the solid lines in Figure 4) and a reduced $\chi^{2}$ of $1.40(p$ value $=17 \%)$. The best-fit intercept $(-3.89)$ is $\approx 0.4$ dex lower than that expected from the H14 model (i.e., -3.48 ; shown as the dotted lines in Figure 4); possible reasons are explained in Sections 3.3 and 3.5.1. Our intercept is also similar to the value $(\approx-3.6)$ derived from Trump et al. (2015), which is based on optically selected AGNs in the local universe $(z<0.1) .{ }^{26}$ In general, X-ray emission from XRBs (the dashed lines) is lower compared to that from AGNs, and it is less significant at high

\footnotetext{
${ }^{24}$ Without this constraint, we can only extend the SFR and $M_{*}$ ranges by $\approx 0.5$ dex (i.e., one bin; see Figures 4 and 5). Such extended samples have very large uncertainties on $\langle\mathrm{BHAR}\rangle(\sim 1$ dex or only upper limit available) likely caused by statistical fluctuations, since each sample has only $\lesssim 20$ sources.

25 We employ the Python code, scipy.optimize.curve_fit, to performing the fitting. We use the median SFR of each bin when we perform the fitting. We adopt the mean values of $1 \sigma$ upper and lower uncertainties on $\langle\mathrm{BHAR}\rangle$ in the fitting. We do not apply an error to the median SFR for simplicity, since the SFR distribution in each bin usually has a strong non-Gaussian shape. The code estimates the uncertainties on best-fit parameters from the covariance matrix. Median SFR and mean SFR are very close, since sources in each bin have similar SFR. Using the median or mean does not affect our results significantly. ${ }^{26}$ Starting from $\lambda_{\text {Edd }} / \mathrm{sSFR} \approx 10^{-2.3}$ in their Figure 18 (where $\lambda_{\text {Edd }}$ is Eddington ratio and SSFR is specific SFR), we obtain BHAR/SFR $\sim 10^{-3.6}$ with the assumption of $\epsilon \sim 0.1$ (Section 2.3) and $M_{*} / M_{\mathrm{BH}} \sim 500$ (e.g., Häring \& Rix 2004; Kormendy \& Ho 2013).
}

SFR. The stacked X-ray emission for individually undetected galaxies is consistent with being entirely due to XRBs.

\subsection{BHAR Dependence on $M_{*}$}

To investigate the relation between $\langle\mathrm{BHAR}\rangle$ and $M_{*}$, we bin our sources in $M_{*}$ and calculate $\langle\mathrm{BHAR}\rangle$ for each bin. The total number of sources is 13,114 (Table 1). ${ }^{27}$ The results are shown in Figure 5. In general, $\langle\mathrm{BHAR}\rangle$ is higher in more massive galaxies, and the fraction of X-ray detected sources rises toward higher $M_{*}$, consistent with previous work (see Section 1). In the high- $z$ bins, $\mathrm{X}$-ray emission from AGNs is comparable to that expected from XRBs for galaxies with $M_{*}<10^{10} M_{\odot}$, but AGN emission becomes dominant for more massive galaxies. The fact that more massive galaxies have higher $\langle\mathrm{BHAR}\rangle$ is also supported by Figures 1 and 2, which demonstrates that most X-ray detections occur in galaxies with $M_{*} \gtrsim 10^{10} M_{\odot}$ despite the fact that those massive galaxies are only $\approx 10 \%$ of the whole population. Similar to the behavior in the left panels of Figure 4, X-ray emission from stacked sources is generally comparable to that expected from XRBs. There are several bins $\left(M_{*} \lesssim 10^{9} M_{\odot}\right)$ with only a few $\mathrm{X}$-ray detected sources $(\lesssim 10$; see Figure 5, right panels). In those bins, the $\left\langle L_{X}\right\rangle$ contribution from X-ray detected sources does not dominate over that from stacked sources; thus, the small numbers of detected sources do not cause large statistical fluctuations.

As in Section 3.1, we perform a linear fitting to the $\langle$ BHAR $\rangle-$ $M_{*}$ relation. If the slope is allowed to vary, we obtain

$$
\log (\langle\mathrm{BHAR}\rangle)=(1.10 \pm 0.08) \log \left(M_{*}\right)-(14.0 \pm 0.8),
$$

with reduced $\chi^{2}=0.88(p$-value $=54 \%)$; if the slope is fixed to unity, we obtain

$$
\log (\langle\mathrm{BHAR}\rangle)=\log \left(M_{*}\right)-(13.0 \pm 0.1),
$$

with reduced $\chi^{2}=0.94$ ( $p$-value $=50 \%$ ). Therefore, as for the $\langle\mathrm{BHAR}\rangle-\mathrm{SFR}$ relation, the $\langle\mathrm{BHAR}\rangle-M_{*}$ relation can also be described acceptably by a linear relation with a slope of unity. From the left panels of Figure 5 , the $\langle\mathrm{BHAR}\rangle-M_{*}$ relation is similar in both redshift ranges. The weak redshift dependence is consistent with the behaviors of the AGN X-ray luminosity function (XLF) and stellar mass function (SMF). Both XLF and SMF of our studied regimes (i.e., $L_{\mathrm{X}} \lesssim 10^{44} \mathrm{erg} \mathrm{s}^{-1}$ and $10^{8} M_{\odot} \lesssim M_{*} \lesssim 10^{11} M_{\odot}$, respectively) drop slightly by $\approx 0.2$ dex from $z=0.8$ to 1.7 , where these redshift values are the medians of the low $z$ and high- $z$ samples, respectively (see, e.g., Tomczak et al. 2014; Ueda et al. 2014).

In the right panels of Figure 5, the median SFR values for the bins with $M_{*} \lesssim 10^{10} M_{\odot}$ are close to those expected from the star-forming main sequence in the model of Behroozi et al. (2013, hereafter B13). For massive galaxies with $M_{*} \gtrsim 10^{10} M_{\odot}$, our median SFRs are systematically lower than the values expected from the star-forming main sequence. This is likely due to the existence of massive evolved systems in our sample. In fact, after removing the quiescent population, our median SFRs agree much better with the B13 model (see Appendix A). For both the B13 model and our data, the SFR- $M_{*}$ relation bends at $M_{*} \gtrsim 10^{10} M_{\odot}$ (Figure 5, right panels), likely due to the depletion of cold gas commonly found in high- $M_{*}$ systems (e.g., Peng et al. 2015).

\footnotetext{
27 The sample size here is different from that in Section 3.1, because the sample here is defined as $10^{8} M_{\odot} \leqslant M_{*}<10^{11} M_{\odot}$ while the sample in Section 3.1 is defined as $0.1 M_{\odot} \leqslant \mathrm{SFR}<100 M_{\odot} \mathrm{yr}^{-1}$.
} 

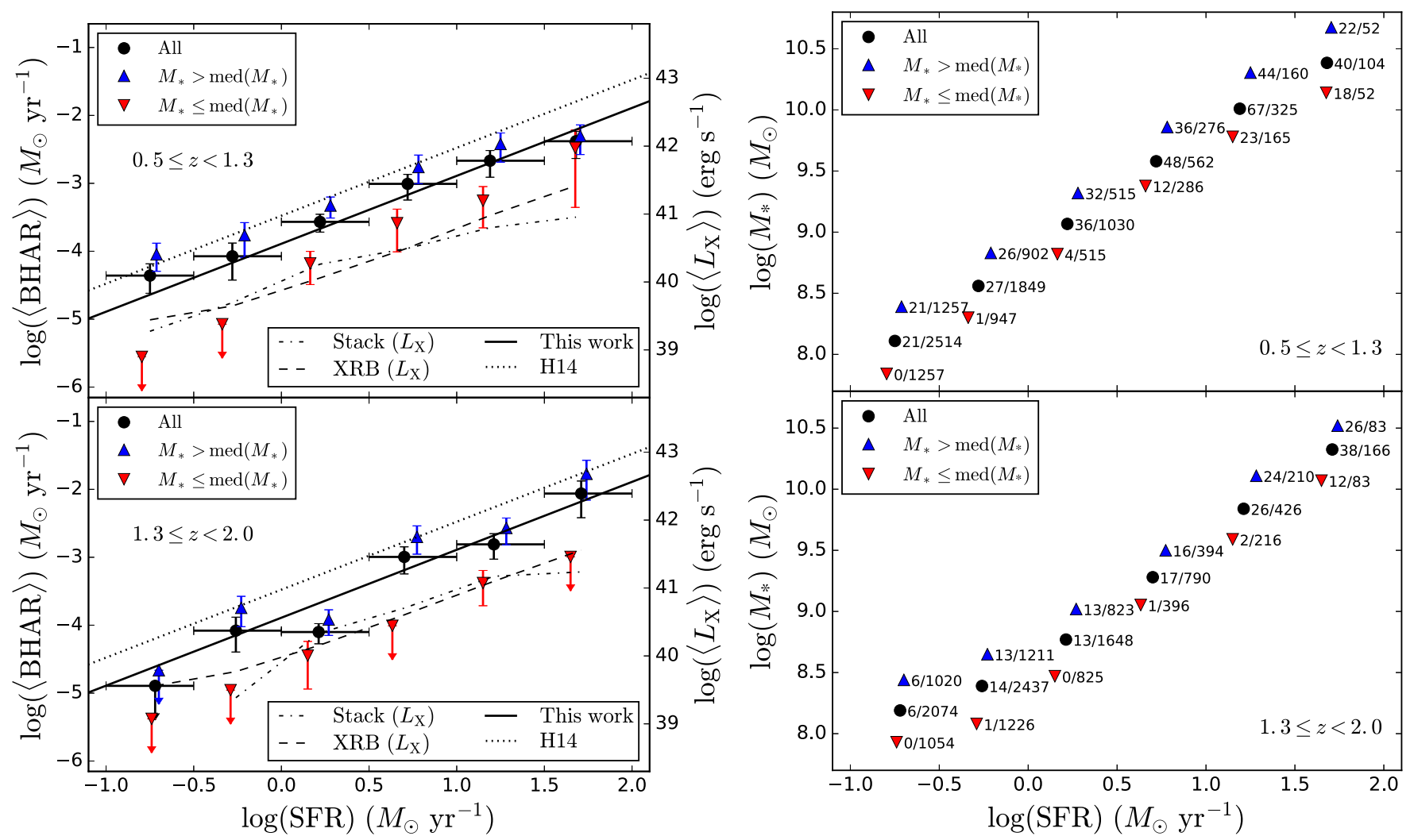

Figure 4. Left panels: $\langle\mathrm{BHAR}\rangle$ as a function of SFR for different redshift ranges. The vertical and horizontal positions of the black points indicate $\langle\mathrm{BHAR}\rangle$ and median SFR, respectively; the horizontal error bars indicate the bin width. The blue upward-pointing and red downward-pointing triangles indicate $\langle$ BHAR $\rangle$ for two subsamples with $M_{*}>\operatorname{med}\left(M_{*}\right)$ and $M_{*} \leqslant \operatorname{med}\left(M_{*}\right)$, respectively, where $\operatorname{med}\left(M_{*}\right)$ is the median $M_{*}$ of sources in each bin (Section 3.3). The solid black line indicates our best-fit linear model with slope fixed at unity; the dotted black line indicates the model proposed by H14. The values of $\langle\mathrm{BHAR}\rangle$ are converted from $\left\langle L_{\mathrm{X}}\right\rangle$, which is labeled on the right side of each panel. $\left\langle L_{X}\right\rangle$ is derived considering both X-ray detected and undetected (via stacking) sources, and contamination from galaxies is subtracted (see Section 2.3). All the errors are estimated via bootstrapping (1000 simulations). If the resulting $1 \sigma$ lower limit of $\left\langle L_{X}\right\rangle$ is negative, we use the $\left\langle L_{\mathrm{X}}\right\rangle$ expected from XRB emission as an upper limit. The dashed black line indicates the $\left\langle L_{\mathrm{X}}, \mathrm{XRB}\right\rangle$ that has been subtracted for each sample. The dotted-dashed line indicates average X-ray luminosities for stacked sources (XRB contributions not subtracted), and it is generally comparable to $\left\langle L_{\mathrm{X}, \mathrm{XRB}}\right\rangle$. In the bottom panel, the lowest-SFR bin has negative stacked $\left\langle L_{\mathrm{X}}\right\rangle$ owing to weak X-ray signals from the stacked sources and background fluctuations; thus, the dotted-dashed line does not extend to the bin with the lowest SFR. Our results can be fitted acceptably by a linear relation between $\langle$ BHAR $\rangle$ and SFR, but more massive galaxies generally have higher $\langle$ BHAR $\rangle$ at a given SFR level. Right panels: median $M_{*}$ corresponding to each (sub)sample on the left. The numbers of X-ray detected sources and all sources in each (sub)sample are marked on the right side of the corresponding point.

\subsection{BHAR Dependence on Both SFR and $M_{*}$}

As shown in Sections 3.1 and 3.2, $\langle$ BHAR $\rangle$ has a positive dependence on both SFR and $M_{*}$. However, SFR and $M_{*}$ are not independent properties for star-forming galaxies, which are the major population in our sample. These two properties are positively related to each other via the star formation main sequence (e.g., Elbaz et al. 2011; also see Figure 2 and the right panels of Figures 4 and 5). Therefore, it is possible that $\langle\mathrm{BHAR}\rangle$ is fundamentally correlated with one factor and the observed relation with the other factor is only a secondary effect.

To investigate this possibility, we compare $\langle\mathrm{BHAR}\rangle$ for sources with different $M_{*}$ (SFR) but similar SFR $\left(M_{*}\right)$. Specifically, we split each sample in Section 3.1 into two subsamples, i.e., with $M_{*} \leqslant \operatorname{med}\left(M_{*}\right)$ and $M_{*}>\operatorname{med}\left(M_{*}\right)$, respectively, where med $\left(M_{*}\right)$ is the median $M_{*}$ in each sample. For completeness, we also split the samples with highest SFR, although the resulting subsamples have less than 100 sources (but more than 50). The high- $M_{*}$ subsamples have similar typical redshifts compared to the corresponding low- $M_{*}$ subsamples; the differences between their median redshifts are $\approx 0.1$. We then calculate $\langle\mathrm{BHAR}\rangle$ for both subsamples (red downward-pointing and blue upward-pointing triangles in
Figure 4). In general, the high- $M_{*}$ subsample has significantly higher $(\approx 0.5-1.5$ dex $)\langle$ BHAR $\rangle$ than its low $-M_{*}$ counterpart. The typical difference between the median $M_{*}$ of the two subsamples is $\approx 0.5$ dex.

Similarly, we also divide each sample in each $M_{*}$ bin (Section 3.2) into two subsamples with SFR $\leqslant \operatorname{med}(\mathrm{SFR})$ and SFR $>$ med(SFR) (Figure 5). The high-SFR subsamples have slightly higher median redshifts than their low-SFR counterparts; the differences are $\approx 0.25$ and 0.1 in the low- $z$ and high$z$ ranges, respectively. This reflects the cosmic evolution of the star-forming main sequence, i.e., galaxies tend to have higher specific SFR (sSFR, defined as SFR $/ M_{*}$ ) at higher redshift.

As shown in Figure 5, in the low- $z$ range, the high-SFR subsample generally has higher $\langle\mathrm{BHAR}\rangle$, but for galaxies in the high $-z$ range, the $\langle$ BHAR $\rangle$ is similar for the two subsamples. Therefore, our results suggest that, at $1.3 \leqslant z<2.0, M_{*}$ is likely to be the main physical property correlated with $\langle$ BHAR $\rangle$, and the observed $\langle$ BHAR $\rangle-$ SFR relation might be a secondary effect caused by the SFR- $M_{*}$ correlation. This is also demonstrated by the comparison between different (sub) samples in the bottom panels of Figure 5. For example, the high-SFR subsample with $10^{9.5} M_{\odot}<M_{*}<10^{10} M_{\odot}$ has median SFR comparable to those of the two samples with highest $M_{*}$, but its $\langle\mathrm{BHAR}\rangle$ value is much lower than those of 

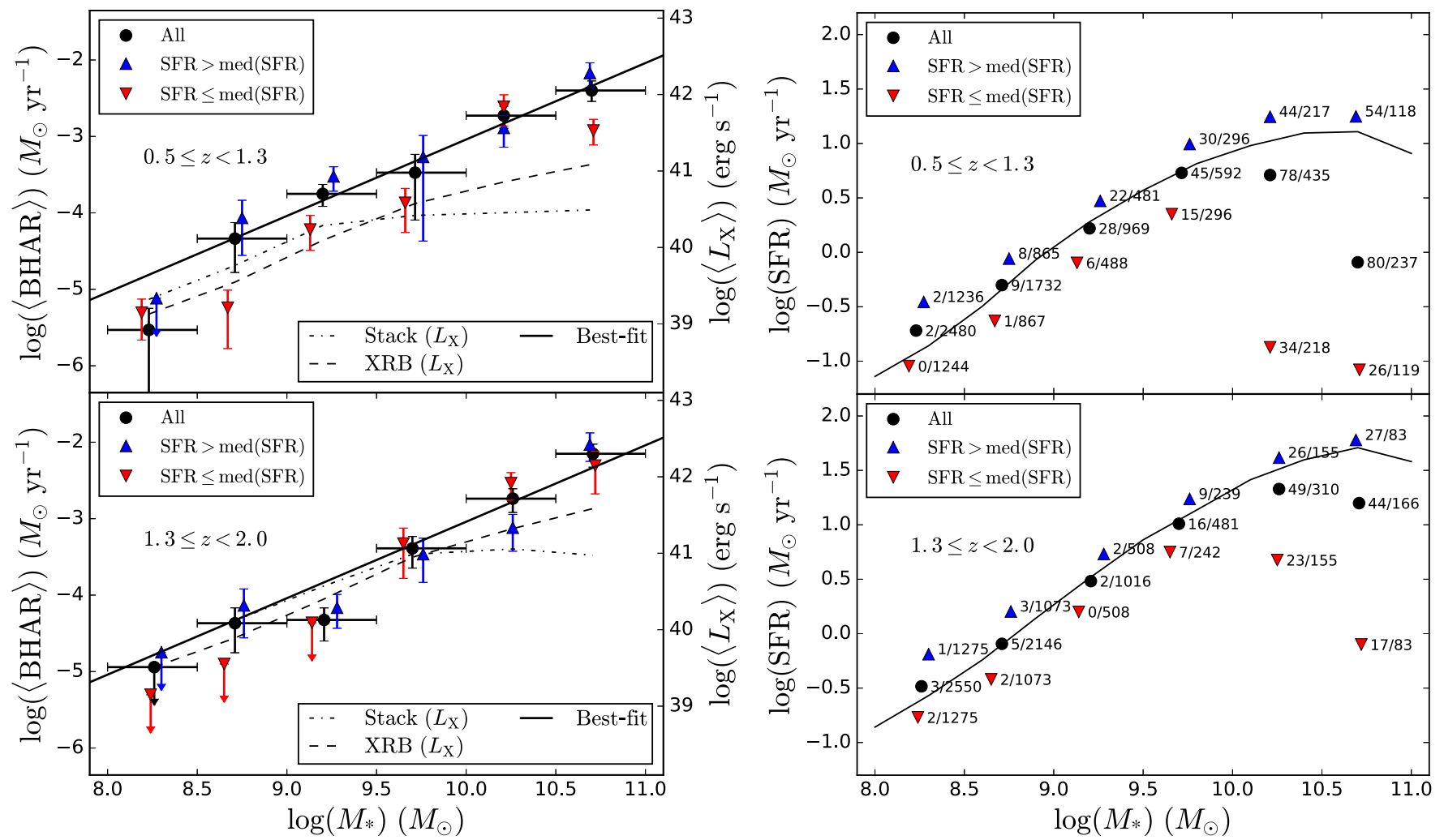

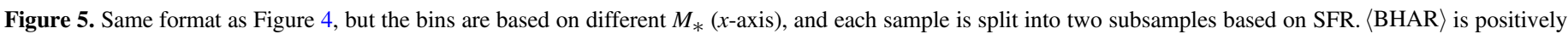
correlated with $M_{*}$. The solid curves in the right panels indicate the star-forming main sequence (B13).

the latter. Nevertheless, we cannot rule out possible correlation between $\langle$ BHAR $\rangle$ and SFR in the high- $z$ range. This is because for bins with $M_{*}<10^{10} M_{\odot}$, the $\left\langle L_{\mathrm{X}}\right\rangle$ from AGNs is low and comparable to $\left\langle L_{\mathrm{X}}, \mathrm{XRB}\right\rangle$. Considering the uncertainties associated with, e.g., stacking procedures and XRB modeling, our data are not sufficiently sensitive to differentiate possible $\langle$ BHAR $\rangle$ differences between high-SFR and low-SFR samples in the low- $\langle\mathrm{BHAR}\rangle$ regime (when X-ray emission from AGNs is comparable to or weaker than that from XRBs). It is thus possible that SFR correlates more strongly with $\langle\mathrm{BHAR}\rangle$ at $M_{*} \gtrsim 10^{10.5} M_{\odot}$, especially in the low- $z$ range (see the rightmost $M_{*}$ bins in the left panels of Figure 5); the dependence of $\langle\mathrm{BHAR}\rangle$ on SFR in the high- $M_{*}$ regime is also suggested by some previous studies (e.g., Delvecchio et al. 2015; Rodighiero et al. 2015; Trump et al. 2015). Rosario et al. (2013) suggest that among massive galaxies with $M_{*} \gtrsim 10^{10.5} M_{\odot}$, X-ray AGNs are more prevalent in high-SFR systems (see also, e.g., Azadi et al. 2015). This is consistent with our observations. From the right panels of Figure 5, in the bins with $10^{10.5} M_{\odot} \leqslant M_{*}<10^{11} M_{\odot}$, the high-SFR subsamples have $\approx 1.5-2$ times higher fractions of X-ray detected sources than the low-SFR subsamples.

To clarify better whether SFR or $M_{*}$ is more important in the low- $z$ range, we bin our sources with $0.5 \leqslant z<1.3$ over grids of $\operatorname{SFR}\left(0.1 M_{\odot} \leqslant \mathrm{SFR}<100 M_{\odot} \mathrm{yr}^{-1}\right)$ and $M_{*}\left(10^{8} M_{\odot} \leqslant\right.$ $\left.M_{*}<10^{11} M_{\odot}\right)$, with the number of sources totaling 5224 (see Table 1). We enlarge the bin width to 1 dex to include more sources in each bin and reduce the uncertainties of $\langle$ BHAR $\rangle$ measurements (see Figure 2). The larger bin width also makes our results less sensitive to the measurement errors on SFR and $M_{*}$. The results are shown in the top panel of
Figure 6. As expected, both SFR and $M_{*}$ display positive correlations with $\langle$ BHAR $\rangle$.

We perform partial correlation (PCOR) analyses on the results using "PCOR" in $\mathrm{R}^{28}$ The PCOR analyses are deployed to measure the correlation between $\langle\mathrm{BHAR}\rangle$ and $\operatorname{SFR}\left(M_{*}\right)$ while controlling for the effects of $M_{*}$ (SFR). There are three statistics available in PCOR to perform the analyses: one parametric statistic (Pearson) and two nonparametric statistics (Spearman and Kendall). We perform the analyses with all three methods and list the $p$-values from each method in Table 2. All $p$-values for the $\langle\mathrm{BHAR}\rangle-M_{*}$ relation are significantly smaller than the corresponding $p$-values for the $\langle$ BHAR $\rangle-$ SFR relation, indicating that $\langle$ BHAR $\rangle$ correlates with $M_{*}$ more strongly than SFR. The parametric method produces $p$-values generally smaller than the nonparametric methods. This is because the parametric method assumes linear relations (see Equations (3)-(6); see also Equations (7) and (8) below) and uses the input data quantitatively, while the nonparametric methods do not have such assumptions but only use ranks of the input data. Among the nonparametric methods, Spearman's statistic leads to more significant relations than Kendall's statistic. The reason is likely that the former uses the value of the difference between two ranks, while the latter is even more conservative and only considers the sign of the difference (e.g.,

\footnotetext{
28 The R code PCOR is available from http://www.yilab.gatech.edu/pcor.html (e.g., Johnson \& Wichern 2002). In the analyses, we provide PCOR with $\log (\langle\mathrm{BHAR}\rangle), \log (\mathrm{SFR})$, and $\log \left(M_{*}\right)$ for sources in each bin (as indicated by the black plus signs in Figure 6). Here, we use a logarithmic scale instead of a linear scale to reduce potential power-law relations to linear relations among the three quantities.
} 

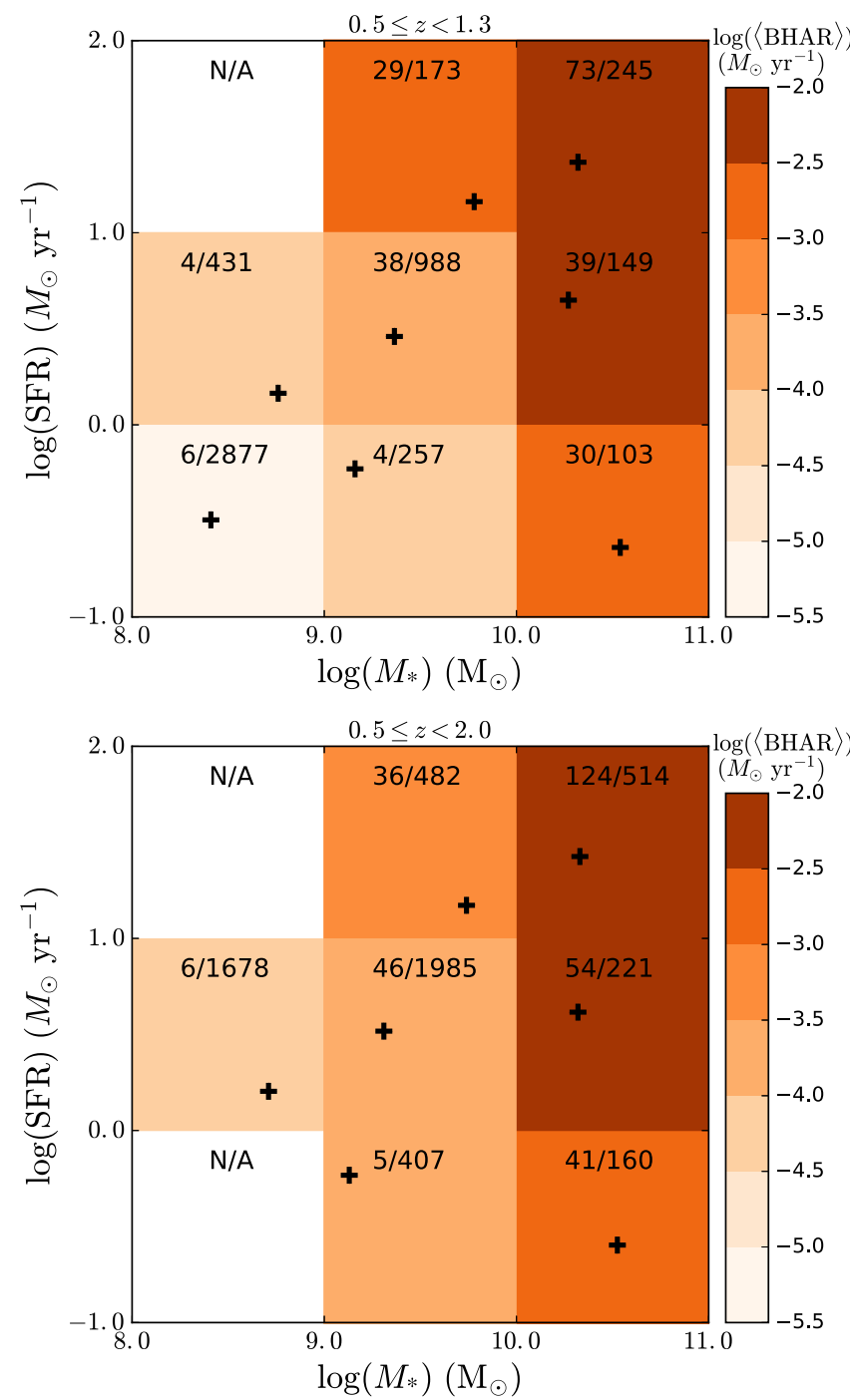

Figure 6. Color-coded $\langle$ BHAR $\rangle$ as a function of SFR and $M_{*}$ at $0.5 \leqslant z<1.3$ (top panel) and $0.5 \leqslant z<2.0$ (bottom panel). The text in the center of each square indicates the number of $\mathrm{X}$-ray detected sources and all sources. The black plus sign indicates median values of SFR and $M_{*}$ for sources in each bin, which are adopted in the analyses in Section 3.3. In both panels, the upper left squares with white color include too few sources (fewer than 100; see Section 3.1), and their $\langle$ BHAR $\rangle$ values are not calculated. In the bottom panel, the $\langle$ BHAR $\rangle$ of the lower left square has a negative $1 \sigma$ lower limit; its value is not shown. The results for the two redshift bins are similar. Both $M_{*}$ and SFR have positive correlations with $\langle\mathrm{BHAR}\rangle$.

Feigelson \& Babu 2012). A linear regression results in

$$
\begin{aligned}
\log (\langle\text { BHAR }\rangle)= & (0.26 \pm 0.11) \log (\text { SFR }) \\
& +(1.24 \pm 0.11) \log \left(M_{*}\right)-(15.5 \pm 1.1)
\end{aligned}
$$

with reduced $\chi^{2}=0.51$ (model-rejection $p$-value $=77 \%$; see footnote 25 for the fitting method).

We also perform the same analyses for sources in both the low $-z$ and high- $z$ ranges together $(11,460$ sources with $0.5 \leqslant z<2.0$ as listed in Table 1). ${ }^{29}$ Figure 6 (bottom panel) displays the results. The $p$-values from PCOR analyses are

\footnotetext{
${ }^{29}$ We do not analyze the high- $z$ range independently, because this would lead to only three available bins having $>100$ sources (see Section 3.1) and wellconstrained $L_{\mathrm{X}}$ (positive $1 \sigma$ lower limit); such a small number of bins is not suitable for PCOR analyses.
}

Table 2

\begin{tabular}{|c|c|c|c|}
\hline \multicolumn{4}{|c|}{$0.5 \leqslant z<1.3$} \\
\hline Relation & Pearson & Spearman & Kendall \\
\hline BHAR-SFR & $10^{-6}(4.8 \sigma)$ & $0.01(2.6 \sigma)$ & $0.34(0.9 \sigma)$ \\
\hline BHAR $-M_{*}$ & $10^{-105}(22 \sigma)$ & $10^{-12}(7.1 \sigma)$ & $0.01(2.6 \sigma)$ \\
\hline \multicolumn{4}{|c|}{$0.5 \leqslant z<2.0$} \\
\hline Relation & Pearson & Spearman & Kendall \\
\hline BHAR-SFR & $0.30(1.0 \sigma)$ & $0.23(1.2 \sigma)$ & $0.68(0.4 \sigma)$ \\
\hline $\mathrm{BHAR}-M_{*}$ & $10^{-14}(7.5 \sigma)$ & $10^{-4}(3.5 \sigma)$ & $0.06(1.9 \sigma)$ \\
\hline
\end{tabular}

$p$-values (Significances) of Partial Correlation Analyses

shown in Table 2 . The $\langle$ BHAR $\rangle-M_{*}$ relation is still significant, in qualitative agreement with the results for the low- $z$ range, but the $\langle$ BHAR $\rangle$-SFR relation becomes insignificant. To visualize the PCOR analyses, we first fit $\langle\mathrm{BHAR}\rangle$ as a linear function of $M_{*}$ (SFR) in logarithmic space, using the same data as in the PCOR analyses (see Figure 6, bottom panel). Then we model the residuals as a linear function of SFR $\left(M_{*}\right)$ and show the results in Figure 7. The resulting residual-SFR relation is flat, and its slope is consistent with zero at a $3 \sigma$ confidence level. However, the residual $-M_{*}$ relation is steep. Therefore, $\langle\mathrm{BHAR}\rangle$ can largely be described via the relation with $M_{*}$ rather than SFR. Similar analyses have also been performed for the lowredshift bin, and the conclusion is the same.

The linear-fitting ( $\langle\mathrm{BHAR}\rangle$ as a function of SFR and $M_{*}$ ) result is

$$
\begin{aligned}
\log (\langle\mathrm{BHAR}\rangle)= & (0.22 \pm 0.08) \log (\mathrm{SFR}) \\
& +(1.16 \pm 0.09) \log \left(M_{*}\right)-(14.6 \pm 0.8)
\end{aligned}
$$

similar to that in the low- $z$ range. The best-fit reduced $\chi^{2}$ is 2.0 , which corresponds to a $p$-value $=9 \%$. The $p$-value is much smaller than the previous value because the errors on $\langle$ BHAR $\rangle$ from $0.5 \leqslant z<2.0(0.10-0.44$ dex with median $=0.14$ dex $)$ are generally smaller than those from $0.5 \leqslant z<1.3(0.15-0.48$ dex with median $=0.22 \mathrm{dex}$ ), due to the increase of sample size in each bin (Figure 6).

The above analyses are based on samples including both star-forming and quiescent galaxies, with star-forming galaxies being the major population $(\approx 80 \%)$. To test whether our main conclusion ( $\langle\mathrm{BHAR}\rangle$ mainly relates to $M_{*}$ ) applies for starforming galaxies alone, we repeat the above analyses with the sample of galaxies near the star-forming main sequence in Appendix A. Our analyses there show that $\langle\mathrm{BHAR}\rangle$ still correlates with $M_{*}$ more strongly than SFR for star-forming galaxies.

\subsection{BHAR/SFR Ratio as a Function of $M_{*}$}

The ratio BHAR/SFR represents the relative growth between SMBHs and their host galaxies, and thus it has important implications for SMBH-galaxy coevolution. To study its dependence on $M_{*}$, we bin our sources based on $M_{*}$ and derive $\langle\mathrm{BHAR}\rangle /\langle\mathrm{SFR}\rangle$ for each bin. The results are displayed in Figure 8. For both redshift ranges, massive galaxies with $M_{*} \gtrsim 10^{10} M_{\odot}$ generally have higher $\langle\mathrm{BHAR}\rangle /\langle\mathrm{SFR}\rangle$. This is understandable considering that $\langle\mathrm{BHAR}\rangle / M_{*}$ is roughly a constant (Section 3.2), while sSFR generally drops for massive galaxies (Figure 5, right panels; also see, e.g., Whitaker et al. 2012; Pan et al. 2017). 

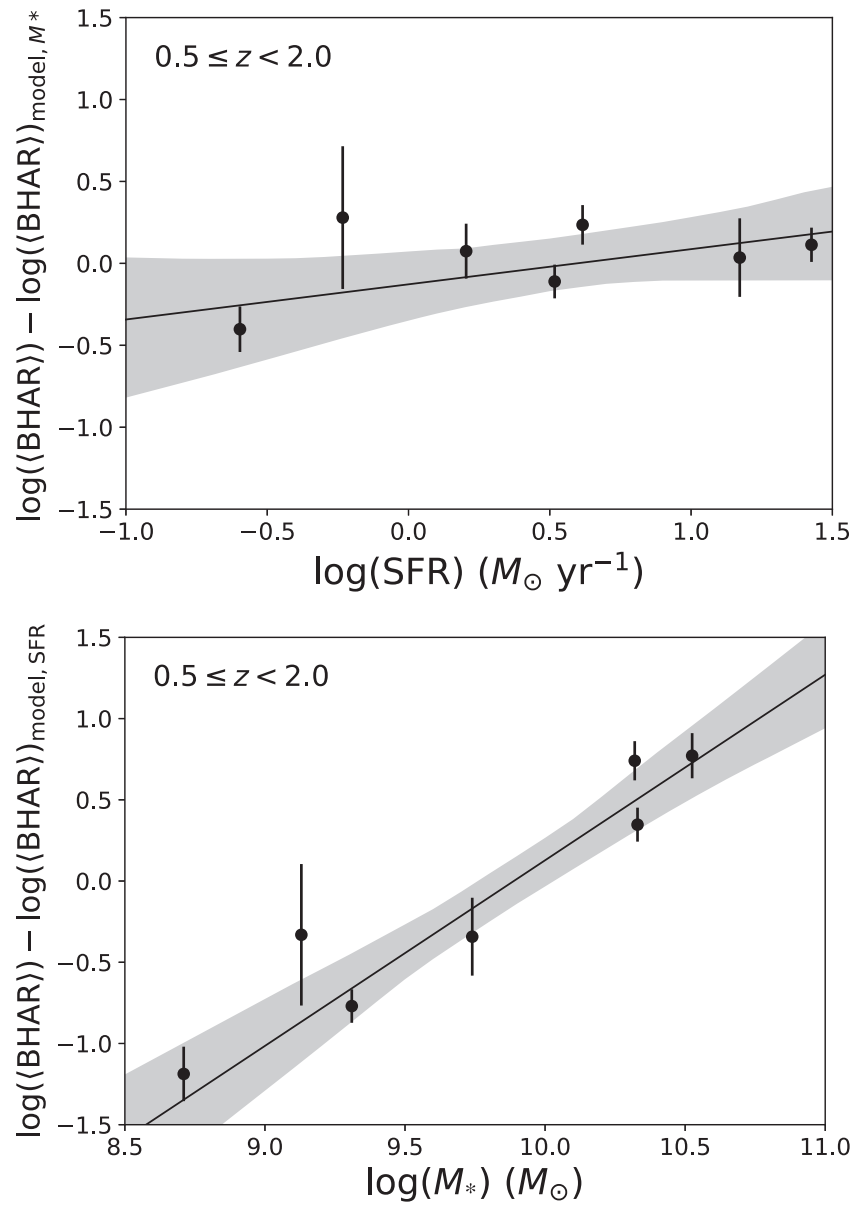

Figure 7. Top: $\langle\mathrm{BHAR}\rangle$ residuals of the $\langle\mathrm{BHAR}\rangle-M_{*}$ fit as a function of SFR. The black solid line indicates the best fit for the residuals as a function of SFR. The shaded region indicates $3 \sigma$ uncertainties derived from Markov chain Monte Carlo sampling utilizing "emcee" (Foreman-Mackey et al. 2013). The residual-SFR relation is flat. Bottom: $\langle\mathrm{BHAR}\rangle$ residual of the $\langle\mathrm{BHAR}\rangle-\mathrm{SFR}$ fit as a function of $M_{*}$. The residual- $M_{*}$ relation is steep.

However, the $\langle\mathrm{BHAR}\rangle /\langle\mathrm{SFR}\rangle$ dependence on $M_{*}$ is not observed by Mullaney et al. (2012b; see the blue open points in Figure 8). This difference is likely because their sample consists of only massive galaxies in a narrow range of $M_{*}$ $\left(10^{10} M_{\odot} \lesssim M_{*} \lesssim 10^{11} M_{\odot}\right)$ and their uncertainties are relatively large. A recent study by Rodighiero et al. (2015), based on galaxies with $10^{10} M_{\odot} \lesssim M_{*} \lesssim 10^{11.5} M_{\odot}$, found that a positive correlation still exists between $\langle\mathrm{BHAR}\rangle /\langle\mathrm{SFR}\rangle$ and $M_{*}$, due to their small error bars on $\langle\mathrm{BHAR}\rangle /\langle\mathrm{SFR}\rangle$. Our $\langle\mathrm{BHAR}\rangle /\langle\mathrm{SFR}\rangle$ values for galaxies having similar $M_{*}$ are slightly lower than those measured in the two studies, likely due to different sample selections and/or the missed accretion power from broad-line AGNs in our analyses (Sections 2.1 and 3.5.1). Indeed, if we assume that the luminous broad-line AGNs have $10^{10} \leqslant M_{*}<10^{11} M_{\odot}$ and include them in our sample, our $\langle$ BHAR $\rangle /\langle\mathrm{SFR}\rangle$ values would be consistent with those in previous studies (see Section 3.5.1 for details). The $M_{*}$-dependent ratio of $\langle\mathrm{BHAR}\rangle /\langle\mathrm{SFR}\rangle$ provides a possible explanation for the fact that our best-fit intercept of the $\langle\mathrm{BHAR}\rangle-\mathrm{SFR}$ relation is lower than that expected from the H14 model (see Section 3.1). The data used by H14 to estimate the $\langle$ BHAR $\rangle-S F R$ relation are mainly for massive galaxies. Since massive galaxies have higher $\langle\mathrm{BHAR}\rangle /\langle\mathrm{SFR}\rangle$ values, the resulting intercept of the $\langle$ BHAR $\rangle-S F R$ relation

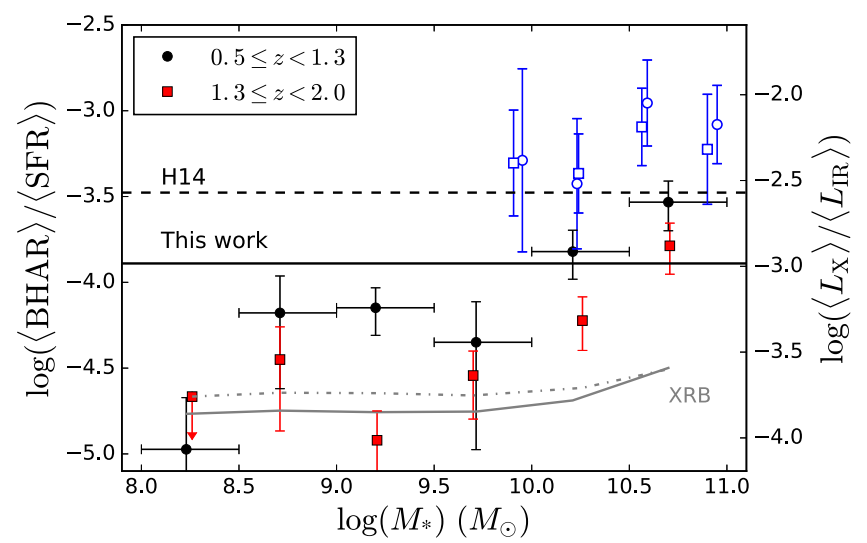

Figure 8. Ratio of $\langle\mathrm{BHAR}\rangle$ to $\langle\mathrm{SFR}\rangle$ as a function of $M_{*}$. The black points and red squares indicate sources in the low $z$ and high- $z$ ranges, respectively. Their horizontal positions indicate median $M_{*}$ in the bin, and the horizontal error bars (only shown for the low $z$ bin) indicate the bin width. The blue open symbols indicate results from Mullaney et al. (2012a); the circles and squares indicate their $z \sim 1$ and $z \sim 2$ samples, respectively. The scale of $\left\langle L_{X}\right\rangle /\left\langle L_{\mathrm{IR}}\right\rangle$ is shown on the right side, where we convert SFR to $L_{\mathrm{IR}}$ with a constant factor (C13). The gray solid and dotted-dashed lines indicate the $\left\langle L_{\mathrm{X}, \mathrm{XRB}}\right\rangle /\left\langle L_{\mathrm{IR}}\right\rangle$ ratios in the low $-z$ and high $-z$ ranges, respectively. These values are used as upper limits for $\left\langle L_{\mathrm{X}}\right\rangle /\left\langle L_{\mathrm{IR}}\right\rangle$ for AGNs, when $1 \sigma$ lower limits of $\left\langle L_{\mathrm{X}}\right\rangle$ for AGNs are negative. The solid horizontal line indicates the intercept of our best-fit $\langle$ BHAR $\rangle-$ SFR linear relation in Figure 4; the dashed horizontal line indicates the H14 model. The $\langle\mathrm{BHAR}\rangle /\langle\mathrm{SFR}\rangle$ ratio is strongly dependent on $M_{*}$. More massive galaxies generally have higher $\langle\mathrm{BHAR}\rangle /\langle\mathrm{SFR}\rangle$, indicating that they are more efficient in growing their SMBHs. For high-mass galaxies $\left(M_{*} \gtrsim 10^{10} M_{\odot}\right)$, our $\langle\mathrm{BHAR}\rangle /\langle\mathrm{SFR}\rangle$ values are systematically lower than those in Mullaney et al. (2012a). This is likely caused by the exclusion of broad-line AGNs in our sample (see Sections 2.1 and 3.5.1).

should be higher. For example, if we only use the high- $M_{*}$ subsamples (blue points in Figure 4) to derive the $\langle$ BHAR $\rangle-$ SFR relation with unity slope, we would obtain a higher intercept.

The $\langle\mathrm{BHAR}\rangle /\langle\mathrm{SFR}\rangle$ ratio in the high- $z$ range is generally lower than that in the low- $z$ range (Figure 8). This is consistent with global AGN activity and star formation studies. The emissivity of AGNs in our luminosity regime $\left(L_{\mathrm{X}} \lesssim 10^{44} \mathrm{erg} \mathrm{s}^{-1}\right.$; see Figure 1$)$ slightly increases from $z \approx 2$ to $z \approx 1$ (e.g., Ueda et al. 2014); meanwhile, the emissivity of star formation drops (e.g., Hopkins 2004 and B13; see also Figure 5, right panels). Physically, the redshift evolution might reflect that, at lower redshift, gas in galaxies is more concentrated in the vicinity of SMBHs.

\subsection{Reliability Checks}

\subsubsection{Missed Accretion Power}

Luminous X-ray emission is almost a universal tracer of SMBH accretion (e.g., Mushotzky 2004; Gibson et al. 2008). Since this work is based on the deepest X-ray survey (the $7 \mathrm{Ms}$ CDF-S) and includes individually undetected sources via stacking analyses (Section 2.3), we are unlikely to miss a large fraction of black hole accretion power due to survey sensitivity (e.g., Brandt \& Alexander 2015). Also, our stacked mean X-ray luminosities are similar to the predicted X-ray emission from XRBs (see Sections 3.1 and 3.2), indicating that most of cosmic accretion power in our redshift range $(0.5 \leqslant z<2.0)$ is directly detected in the 7 Ms CDF-S. Due to the small size of CANDELS/GOODS-South $\left(170 \operatorname{arcmin}^{2}\right)$, our sample will miss AGNs at the bright end of the XLF $\left(L_{\mathrm{X}} \gtrsim 10^{44} \mathrm{erg} \mathrm{s}^{-1}\right.$; see Figure 1). For the CANDELS/ GOODS-South field $\left(170 \operatorname{arcmin}^{2}\right)$, the fraction of missed 
accretion power is $\approx 37 \%$ for $0.5 \leqslant z<2.0$, estimated based on the XLF model of Aird et al. (2010). However, those luminous sources are likely to reside in massive systems $\left(M_{*} \gtrsim 10^{10} M_{\odot}\right.$; e.g., Bahcall et al. 1997; Kauffmann et al. 2003; Matsuoka et al. 2014), and thus the bright-end correction is not likely to boost $\langle\mathrm{BHAR}\rangle$ for low- $M_{*}$ galaxies and affect our main conclusions (i.e., massive galaxies have higher $\langle\mathrm{BHAR}\rangle$ and $\langle\mathrm{BHAR}\rangle /\langle\mathrm{SFR}\rangle$ than less massive ones). Moreover, a recent study of luminous quasars also suggests that their BHAR might be primarily related to $M_{*}$ rather than SFR (Xu et al. 2015).

A large population of CTK AGNs might exist but show almost no X-ray signal, even in the unprecedentedly deep $7 \mathrm{Ms}$ CDF-S (e.g., see Section 3.3 of Brandt \& Alexander 2015; Comastri et al. 2015). Their accretion power would be largely missed in our analyses. We have stacked hard-band (observedframe $2-7 \mathrm{keV}$ ) X-ray images and compared the results with the stacking of the soft band (observed-frame $0.5-2 \mathrm{keV}$ ). We do not find evidence of CTK populations. Nevertheless, it is possible to find signatures of CTK AGNs with more refined analyses (e.g., stacking of very hard narrower bands), and we will perform such analyses in a dedicated paper (B. Luo et al., in preparation). The prevalence of CTK AGNs is predicted by population-synthesis models of the cosmic X-ray background, although these models have significant uncertainties in the regime of very high $N_{\mathrm{H}}$ (e.g., Gilli et al. 2007; Ueda et al. 2014). The predicted total X-ray emission from CTK AGNs is usually less than that from other AGNs (e.g., Gilli et al. 2007; Treister et al. 2009; also see Buchner et al. 2015, for relevant spectral fitting), consistent with the very hard X-ray $(\gtrsim 10 \mathrm{keV}$ ) observations of the local universe (e.g., Akylas et al. 2016; Koss et al. 2016). Even if these CTK AGNs predominantly reside in low- $M_{*}$ systems, the total $L_{\mathrm{X}}$ from these galaxies will be at most the same as that from high- $M_{*}$ galaxies; the average $L_{\mathrm{X}}$ in low- $M_{*}$ galaxies should be still much lower than for massive galaxies, as the former has much larger number density. Their inclusion is unlikely to make qualitative changes to our main conclusion that $\langle\mathrm{BHAR}\rangle$ is a strong function of $M_{*}$. Nevertheless, the possible existence of the CTK AGN population could increase/decrease the dependence on other galaxy properties at given $M_{*}$. Some observations suggest that CTK AGNs are more prevalent in galaxies with high SFRs and/or that are experiencing major mergers (e.g., Juneau et al. 2013; Kocevski et al. 2015), while the dependence on redshift tends not to be strong (e.g., Buchner et al. 2015).

Nineteen broad-line AGNs have been deliberately excluded from our sample (see Section 2.1). The majority of them $(14 / 19)$ are X-ray luminous with $10^{43}<L_{X} \lesssim 10^{44} \mathrm{erg} \mathrm{s}^{-1}$ (see Figure 1, bottom panel). The rarity of broad-line AGNs with $L_{\mathrm{X}}<10^{43} \mathrm{erg} \mathrm{s}^{-1}$ is consistent with, e.g., Merloni et al. (2014). The 14 luminous broad-line AGNs make up 25\% of the total AGN population with $L_{\mathrm{X}}>10^{43} \mathrm{erg} \mathrm{s}^{-1}$. This fraction agrees with Figure 7 of Merloni et al. (2014), which shows that the fraction of broad-line AGNs is $\approx 20 \%-40 \%$ for $10^{43} \lesssim L_{\mathrm{X}} \lesssim 10^{44} \mathrm{erg} \mathrm{s}^{-1}$. Therefore, we are not missing a significant fraction of broad-line AGNs due to, e.g., low-S/N spectra. Those luminous broad-line AGNs are likely to reside in massive galaxies (e.g., Bahcall et al. 1997; Matsuoka et al. 2014). Indeed, $\approx 90 \%$ of our non-broad-line AGNs in the same luminosity regime are hosted by galaxies with $M_{*}>10^{10} M_{\odot}$, and these should be physically similar systems to broad-line AGNs following the standard unified AGN model. Hence, including the broad-line AGNs would boost $\langle\mathrm{BHAR}\rangle$ for massive galaxies and thus would make our main conclusions even stronger. This might also explain why our $\langle\mathrm{BHAR}\rangle /\langle\mathrm{SFR}\rangle$ ratio for massive galaxies is lower than the values from previous studies (see Sections 3.1 and 3.4). Quantitatively, if we include those 14 luminous broad-line AGNs assuming that they reside in galaxies with $10^{10} \leqslant M_{*}<10^{11} M_{\odot}$, the $\langle\mathrm{BHAR}\rangle /\langle\mathrm{SFR}\rangle$ values for this $M_{*}$ bin would be $10^{-3.3}$ and $10^{-3.7}$, respectively, in the low- $z$ and high- $z$ ranges. These values are consistent with previous studies (see Figure 8). Considering the small population and relatively low luminosities $\left(L_{\mathrm{X}}<10^{43} \mathrm{erg} \mathrm{s}^{-1}\right)$ of the remaining five broad-line AGNs, excluding them is not likely to affect our results significantly.

\subsubsection{Sample $M_{*}$ and SFR Completeness}

In the high- $z$ range, our sample is not complete at $M_{*} \sim 10^{7} M_{\odot}$ (Figure 1, top panel), and this is the main reason why this study focuses on galaxies with $M_{*} \gtrsim 10^{8} M_{\odot}$. To check quantitatively whether our sample is complete down to $M_{*} \sim 10^{8} M_{\odot}$ in the high- $z$ range, we calculate the comoving number density for the bin with lowest $M_{*}$ and compare it with the model of B13. There are 2550 sources in the bin (see Figure 5); the comoving volume covered by the CANDELS/GOODS-South field (170 $\operatorname{arcmin}^{2}$; see Guo et al. 2013) is $3.8 \times 10^{5} \mathrm{Mpc}^{3}$. Thus, the comoving number density for our galaxies having $10^{8} M_{\odot} \leqslant M_{*}<10^{8.5} M_{\odot}$ is $1.3 \times 10^{-2} \mathrm{Mpc}^{-3} \mathrm{dex}^{-1}$. This value is roughly consistent with Figure 3 of B13, indicating that our sample is basically complete above $M_{*} \sim 10^{8} M_{\odot}$.

A color-dependent completeness issue might exist in our flux-limited sample (e.g., Xue et al. 2010). This is because for a given $M_{*}$, blue galaxies generally have higher optical-to-nearIR luminosities than red galaxies, due to their relatively young stellar populations. Therefore, flux-limited optical/IR surveys like CANDELS might miss the red population in the low$M_{*}$ regimes. However, our sample is not likely to have this issue, since our SFR- $M_{*}$ relation agrees well with the B13 model at the low- $M_{*}$ end (see the right panels of Figure 5). If our sample were biased to blue (high-SFR) galaxies at the low$M_{*}$ end, the measured SFR would be significantly above the model value.

The fact that our sample generally does not have colordependent completeness is not surprising. At the low- $M_{*}$ $\left(\sim 10^{8} M_{\odot}\right)$ regime that we probe, the spread in rest-frame colors is relatively small, i.e., most low- $M_{*}$ (thus low-luminosity) galaxies reside in the "blue cloud" rather than the "red sequence" in color-magnitude diagrams (e.g., Schneider 2014; Martis et al. 2016; Pan et al. 2017). This is broadly consistent with the right panels of Figure 5, assuming that star-forming activity is generally traced by colors. The SFR dispersion is only $\lesssim 0.3 \mathrm{dex}$ in the bins whose $M_{*} \lesssim 10^{10} M_{\odot}$, i.e., most galaxies are located around the star-forming main sequence. In the high- $M_{*}$ regime where the red sequence exists, our sample is also likely to be complete. This is because the comoving number density is $8.5 \times 10^{-4} \mathrm{Mpc}^{-3} \mathrm{dex}^{-1}$ for the $10^{10.5} M_{\odot} \leqslant M_{*}<10^{11} M_{\odot}$ bin in the high- $z$ range; this value is consistent with the SMF derived in a dedicated study $\left(9.3_{-1.7}^{+1.9} \times 10^{-4} \mathrm{Mpc}^{-3} \mathrm{dex}^{-1}\right.$; see Table 1 of Tomczak et al. 2014), which includes both starforming and quiescent galaxies. 
On the other hand, our lowest-SFR regime $(\mathrm{SFR} \sim$ $0.1 M_{\odot} \mathrm{yr}^{-1}$ ) corresponds to $M_{*} \sim 10^{8} M_{\odot}$ on the starforming main sequence (see the right panels of Figures 4 and 5). Since our sample is basically complete above $M_{*} \sim 10^{8} M_{\odot}$, it should also be roughly complete above SFR $\sim 0.1 M_{\odot} \mathrm{yr}^{-1}$. Moreover, our conclusions do not critically depend on the galaxies with SFR $\sim 0.1 M_{\odot} \mathrm{yr}^{-1}$ and $M_{*} \sim 10^{8} M_{\odot}$. Thus, even if there are minor completeness issues in our lowest-SFR and/or lowest- $M_{*}$ bins, our results should not be affected materially.

\section{Discussion \\ 4.1. SMBH-Galaxy Coevolution}

The linear relation between $\langle\mathrm{BHAR}\rangle$ and SFR was previously derived for observations of high-SFR galaxies (SFR $\gtrsim 10 M_{\odot} \mathrm{yr}^{-1}$; e.g., Symeonidis et al. 2011; C13). For the first time, our results show that this linear relation remains applicable to galaxies with SFRs down to $\sim 0.1 M_{\odot} \mathrm{yr}^{-1}$ in the redshift range of $0.5 \leqslant z<2.0$ (Section 3.1). The relation demonstrates that SMBH and galaxy growth broadly track each other over cosmic time. This is consistent with the observational fact that the evolutions of cosmic BHAR and SFR have broadly similar shapes. The normalization of cosmic SFR relative to cosmic BHAR is $\sim 3.7$ dex (see, e.g., Silverman et al. 2008; Aird et al. 2010; Kormendy \& Ho 2013), similar to our best-fit intercept of the $\langle$ BHAR $\rangle-$ SFR relation.

The linear $\langle$ BHAR $\rangle-$ SFR relation suggests a simple scenario of coevolution. H14 assumed that, for any individual galaxy, the ratio between the amount of gas accreted by its SMBH and that used to form stars is a universal constant (when averaged over $\sim 100 \mathrm{Myr}$ ). Under this assumption, the $\langle$ BHAR $\rangle$ for different samples of similar SFR should be similar. However, this assumption appears in contradiction with our observational results. For a given SFR level, the sources with larger $M_{*}$ have significantly higher $\langle\mathrm{BHAR}\rangle$ (Section 3.3). In addition, $\langle\mathrm{BHAR}\rangle$ is also related to $M_{*}$ linearly (Section 3.2), and $\langle\mathrm{BHAR}\rangle$ is correlated with $M_{*}$ more strongly than SFR as indicated by our PCOR analyses in Section 3.3. Therefore, our results suggest that $\langle\mathrm{BHAR}\rangle$ might be intrinsically linked to $M_{*}$, and this $\langle\mathrm{BHAR}\rangle-M_{*}$ relation and the star-forming main sequence together might largely cause the observed $\langle\mathrm{BHAR}\rangle-\mathrm{SFR}$ relation as a secondary effect.

In the analyses of Section 3.3, we find when controlling for $M_{*}$ that the dependence of $\langle\mathrm{BHAR}\rangle$ on SFR is relatively weak. We have furthermore checked the $\langle$ BHAR $\rangle$-sSFR relation for the whole sample and do not find any significant trend.

\subsection{The Physical Link between BHAR and $M_{*}$}

It has been well established that X-ray-selected AGNs above a given $L_{\mathrm{X}}$ threshold are preferentially found in massive galaxies (e.g., Xue et al. 2010; Brandt \& Alexander 2015). This finding is consistent with our results that $\langle\mathrm{BHAR}\rangle$ depends strongly on $M_{*}$, even for SFR-controlled samples (Sections 3.2 and 3.3). In fact, $M_{*}$, rather than SFR, appears to be the primary factor related to $\langle\mathrm{BHAR}\rangle$.

Massive galaxies with $M_{*} \gtrsim 10^{10} M_{\odot}$ have lower sSFR (Section 3.4) than less massive galaxies. If we assume that SFR reflects the amount of cold gas available, the decrease of SSFR for massive galaxies indicates that the mass fraction of cold gas drops toward high $M_{*}$ (e.g., Saintonge et al. 2016). The cold gas needed for star formation is also likely responsible for fueling black hole accretion (e.g., Alexander \& Hickox 2012; Vito et al. 2014), while hot-gas accretion could power lowluminosity AGNs that generally have little contribution to total black hole growth (see, e.g., Figure 3 of Croton et al. 2006; Heckman \& Best 2014; Yuan \& Narayan 2014). The $M_{*-}$ dependent $\langle\mathrm{BHAR}\rangle /\langle\mathrm{SFR}\rangle$ indicates that the massive population is generally more efficient in feeding cold gas to their SMBHs (Figure 8). This could be further broadly interpreted in two possible respects. First, the black hole fueling efficiency of each galaxy might depend on $M_{*}$, due to several physically plausible causes:

1. The potential wells in galactic centers are deeper for massive galaxies, making it easier for gas particles to fall into the galaxy center and fuel the SMBH. More specifically, supernova feedback might prevent gas from falling into the galaxy center when the potential well is not sufficiently deep (e.g., Bellovary et al. 2013; Dubois et al. 2015).

2. Compared to low- $M_{*}$ systems, high- $M_{*}$ ones are more likely to have bars and major mergers (e.g., Melvin et al. 2014; Rodriguez-Gomez et al. 2015) that could induce gas inflow effectively (e.g., Alexander \& Hickox 2012).

3. Massive galaxies often have more massive SMBHs than low- $M_{*}$ galaxies (e.g., Kormendy \& Ho 2013). Those SMBHs, having a stronger gravitational field, are more capable of accreting gas from their vicinity. In fact, some studies suggest that long-term BHAR is proportional to $M_{\mathrm{BH}}$, resulting from a universal Eddington ratio distribution (e.g., Aird et al. 2012; Jones et al. 2016).

Second, the SMBH occupation fraction might drop toward low $M_{*}\left(M_{*} \lesssim 10^{10} M_{\odot}\right)$, and some low- $M_{*}$ galaxies might only host intermediate-mass black holes (IMBHs) with $M_{\mathrm{BH}} \lesssim 10^{4} M_{\odot}$ (e.g., Volonteri 2010; Miller et al. 2015; Trump et al. 2015). Due to the Eddington limit, the X-ray emission from accretion onto IMBHs is likely to be much weaker than that from SMBHs. The $\langle$ BHAR $\rangle$ could thus be diminished for galaxies with lower $M_{*}$.

\subsection{Implications for the $M_{B H}-M_{*}$ Relation in the Local Universe}

Our results have implications for the $M_{\mathrm{BH}}-M_{*}$ relation in the local universe, and we illustrate this with some basic arguments below. The $M_{\mathrm{BH}} / M_{*}$ ratio for a galaxy at $z=0$ can be estimated as

$$
\frac{M_{\mathrm{BH}}\left(t_{0}\right)}{M_{*}\left(t_{0}\right)} \approx \frac{M_{\mathrm{BH}}\left(t_{2}\right)+\int_{t_{2}}^{t_{0}} \operatorname{BHAR}(t) d t}{M_{*}\left(t_{2}\right)+\int_{t_{2}}^{t_{0}} \operatorname{SFR}(t) d t},
$$

where $t$ is cosmic time and the subscripts of $t$ indicate redshift. Assuming that the mass of SMBH seeds is small compared to that accreted over cosmic history (e.g., Volonteri 2010) and that most black hole growth happens at $z \lesssim 2,{ }^{30}$ we have $M_{\mathrm{BH}}\left(t_{2}\right)+$

\footnotetext{
This is broadly supported by the Sołtan argument, such that $\approx 70 \%-80 \%$ of total black hole accretion (including the contribution from luminous quasars) happens at $z \lesssim 2$ (e.g., Ueda et al. 2014; Brandt \& Alexander 2015). Highredshift luminous quasars mostly form SMBHs above $z \sim 2$ (e.g., Wu et al. 2015; Bañados et al. 2016), but they are rare objects and their discussion is beyond the scope of this study.
} 
$\int_{t_{2}}^{t_{0}} \operatorname{BHAR}(t) d t \approx \int_{t_{2}}^{t_{0}} \operatorname{BHAR}(t) d t$, and Equation (9) can be simplified as

$$
\frac{M_{\mathrm{BH}}\left(t_{0}\right)}{M_{*}\left(t_{0}\right)} \approx \frac{\int_{t_{2}}^{t_{0}} \operatorname{BHAR}(t) d t}{M_{*}\left(t_{2}\right)+\int_{t_{2}}^{t_{0}} \operatorname{SFR}(t) d t} .
$$

For local giant ellipticals $\left(M_{*} \gtrsim 10^{11} M_{\odot}\right)$, most of their stars are likely to have been formed at $z \gtrsim 2$ (e.g., Chiosi \& Carraro 2002; Siudek et al. 2016). Thus, $M_{*}$ is roughly the same over $z \approx 0-2$, i.e., $M_{*} \equiv M_{*}\left(t_{0}\right) \approx M_{*}\left(t_{2}\right)$, and Equation (10) is approximately ${ }^{31}$

$$
\frac{M_{\mathrm{BH}}\left(t_{0}\right)}{M_{*}} \approx \frac{\int_{t_{2}}^{t_{0}} \operatorname{BHAR}(t) d t}{M_{*}} \text { (elliptical). }
$$

If we assume that the BHAR- $M_{*}$ linear relation extends to $M_{*} \gtrsim 10^{11} M_{\odot}$ and has not evolved significantly for $z \lesssim 2$ (Equation (6) and Figure 5), Equation (11) leads to

$$
\begin{aligned}
\frac{M_{\mathrm{BH}}\left(t_{0}\right)}{M_{*}} & \approx \frac{\left(t_{2}-t_{0}\right) \mathrm{BHAR}}{M_{*}} \text { (elliptical) } \\
& \approx 10 \mathrm{Gyr} \times 10^{-13} \mathrm{yr}^{-1} \\
& \approx 10^{-3} .
\end{aligned}
$$

Therefore, the $M_{\mathrm{BH}} / M_{*}$ ratio for giant ellipticals should be approximately a constant $(1 / 1000)$, similar to the value $(\approx 1 / 700)$ observed by Häring \& Rix (2004). Considering that the existence of Compton-thick and broad-line AGNs could boost our observed $\langle\mathrm{BHAR}\rangle$ (see Section 3.5.1), the $M_{\mathrm{BH}} / M_{*}$ ratio might be several times larger and more consistent with the value $(\approx 1 / 300)$ from Kormendy \& Ho (2013; but also see Shankar et al. 2016).

The above argument obviously depends on the assumption that giant ellipticals grow their $M_{\mathrm{BH}}$ mostly at $z \lesssim 2$. This assumption, although under debate, is supported by observations of submillimeter galaxies (SMGs; see, e.g., Section 8.6.7 of Kormendy \& Ho 2013). SMGs are likely the high-redshift $(z \sim 2)$ progenitors of massive ellipticals (e.g., Casey et al. 2014; Toft et al. 2014), and the growth of their SMBHs tends to lag that of the host galaxies (e.g., Borys et al. 2005; Alexander et al. 2008). Nevertheless, it is also possible to reproduce the local $M_{\mathrm{BH}} / M_{*}$ ratio if both the growth of SMBHs and that of host galaxies take place at $z \gtrsim 2$ and have strong interplay. Simulations show that AGN feedback can keep a tight $M_{\mathrm{BH}}-M_{*}$ relation when both SMBHs and host galaxies grow at high redshift (e.g., Volonteri et al. 2016).

For local star-forming galaxies, most of their $M_{*}$ is likely to be assembled at $z \lesssim 2$ (e.g., B13). Thus, we have $M_{*}\left(t_{2}\right)+\int_{t_{2}}^{t_{0}} \operatorname{SFR}(t) d t \approx \int_{t_{2}}^{t_{0}} \operatorname{SFR}(t) d t$, and Equation (10) becomes

$$
\begin{aligned}
\frac{M_{\mathrm{BH}}\left(t_{0}\right)}{M_{*}\left(t_{0}\right)} & \approx \frac{\int_{t_{2}}^{t_{0}} \operatorname{BHAR}(t) d t}{\int_{t_{2}}^{t_{0}} \operatorname{SFR}(t) d t}(\text { star-forming }) \\
& \sim \frac{\mathrm{BHAR}}{\operatorname{SFR}} \sim 10^{-5}-10^{-3.5},
\end{aligned}
$$

\footnotetext{
31 For galaxy mergers, Equation (11) is still correct, provided that mergers increase $M_{\mathrm{BH}}$ and $M_{*}$ proportionally.
}

where we adopt the BHAR/SFR range from Figures 8 and 11. Our results in Section 3.4 show that long-term average BHAR/ SFR positively depends on $M_{*}$, and thus $M_{\mathrm{BH}}\left(t_{0}\right) / M_{*}\left(t_{0}\right)$ is higher for massive galaxies than dwarf galaxies. This prediction is supported by some observations. Miller et al. (2015) and Trump et al. (2015) suggest that dwarf galaxies have a lower black hole occupation fraction than massive galaxies, implying a generally lower $M_{\mathrm{BH}} / M_{*}$ for dwarfs. In addition, for some nearby galaxies (e.g., M33, NGC 205, and NGC 404), studies of nuclear kinematics place tight upper limits on $M_{\mathrm{BH}}$, indicating the absence of SMBHs (e.g., Gebhardt et al. 2001; Valluri et al. 2005; Nguyen et al. 2017).

Equations (12) and (13) suggest that ellipticals generally have higher $M_{\mathrm{BH}} / M_{*}$ ratios than star-forming galaxies, consistent with some recent studies (e.g., Reines \& Volonteri 2015; Greene et al. 2016). From the viewpoint of this study, it is understandable why the observed local $M_{\mathrm{BH}} / M_{*}$ values ( 1/500; e.g., Häring \& Rix 2004; Kormendy \& Ho 2013) are much higher than cosmic BHAR/SFR $(\sim 1 / 5000$; e.g., Silverman et al. 2008; Aird et al. 2010). This is because local $M_{\mathrm{BH}} / M_{*}$ measurements are mainly based on observations of passive ellipticals (Equation (12)), and cosmic BHAR/SFR is generally linked to $M_{\mathrm{BH}} / M_{*}$ of star-forming galaxies (Equation (13)).

From Equation (12), for massive ellipticals, the $M_{\mathrm{BH}} / M_{*}$ is expected to be lower in the early universe $(z \gtrsim 1)$; for starforming galaxies, it should depend on $M_{*}$ and have relatively weak cosmic evolution according to Equation (13). As discussed above, observations of SMGs support this scenario. Some studies of high-redshift quasars find higher $M_{\mathrm{BH}} / M_{*}$ ratios than the local values, not expected in our scheme (e.g., Ho 2007; Merloni et al. 2010; but also see, e.g., Jahnke et al. 2009; Sun et al. 2015). However, the $M_{\mathrm{BH}} / M_{*}$ measured from quasars might be biased and not representative for the majority of galaxies (e.g., Lauer et al. 2007). Also, large uncertainties often exist in the measurements of both $M_{\mathrm{BH}}$ and $M_{*}$ for these quasars (e.g., Bongiorno et al. 2012; Shen 2013).

\section{Summary and Future Prospects}

We have studied the dependence of SMBH growth on the SFR and $M_{*}$ of host galaxies at $0.5 \leqslant z<2.0$. Specifically, we compare $\langle$ BHAR $\rangle$ for samples with different SFR and/or $M_{*}$. Due to the deep multiwavelength data in the CDF-S, we are able to probe black hole accretion in hosts down to $\mathrm{SFR} \sim 0.1 M_{\odot} \mathrm{yr}^{-1}$ and $M_{*} \sim 10^{8} M_{\odot}$ with reasonable completeness. Our main results are summarized below.

1. $\langle$ BHAR $\rangle$ correlates with SFR linearly (Section 3.1). However, for SFR-controlled samples, galaxies with higher $M_{*}$ have higher $\langle\mathrm{BHAR}\rangle$ (Section 3.3). Thus, SFR does not appear to be uniquely related to $\langle$ BHAR $\rangle$. The scenario in which long-term average BHAR is only determined by host-galaxy SFR is over simplified (Section 4.1).

2. $\langle\mathrm{BHAR}\rangle$ is also proportional to $M_{*}$ (Section 3.2). In fact, the correlation between $\langle\mathrm{BHAR}\rangle$ and $M_{*}$ is stronger than that between $\langle\mathrm{BHAR}\rangle$ and SFR, suggesting $M_{*}$ as the primary host-galaxy property related to SMBH growth. This result also holds for the star-forming population alone (Appendix A). The observed $\langle$ BHAR $\rangle-S F R$ correlation might be largely a secondary effect due to 
the existence of the star formation main sequence (Section 4.1).

3. Massive galaxies $\left(M_{*} \gtrsim 10^{10} M_{\odot}\right)$ have higher $\langle\mathrm{BHAR}\rangle /\langle\mathrm{SFR}\rangle$ ratios than their less massive counterparts (Section 3.4), suggesting that they have higher black hole fueling efficiency and/or SMBH occupation fraction (Section 4.2).

4. Our results can naturally explain the observed $M_{\mathrm{BH}}-M_{*}$ relation for local giant ellipticals and indicate that they have higher $M_{\mathrm{BH}} / M_{*}$ than their progenitors in the earlier universe (Section 4.3). Also, our results predict that $M_{\mathrm{BH}} / M_{*}$ for giant ellipticals is higher than that for starforming galaxies in the nearby universe. Among local star-forming galaxies, the $M_{\mathrm{BH}} / M_{*}$ values for massive galaxies are likely to be higher than those for dwarfs.

In the future, this study could be extended to galaxies with larger $M_{*}$ (SFR) by compiling a large number of luminous galaxies (Section 3.1). To perform this investigation, analyses based on multiwavelength surveys of wider fields, e.g., COSMOS, XMM-LSS, and Stripe 82, are needed. In addition, it is possible to extend this study to higher redshift using the CDF-S field, but this approach will require SED fitting that can eliminate potential high-redshift biases for SFR and $M_{*}$ measurements (Section 2.2). It would also be worthwhile to derive quantitative black hole fueling efficiency and/or SMBH occupation fraction estimates as a function of $M_{*}$, based on the $M_{*}$-dependent $\langle\mathrm{BHAR}\rangle /\langle\mathrm{SFR}\rangle$ (Section 3.4). Future work could study the BHAR for giant ellipticals by including morphological information (Section 4.3) and the connection between BHAR and host-galaxy gas content by using ALMA observations.

We thank the referee for helpful feedback that improved this work. We thank Robin Ciardullo, Ryan Hickox, Luis Ho, Paola Santini, and Xue-Bing Wu for helpful discussions. G.Y., C.-T. J.C., F.V., and W.N.B. acknowledge financial support from CXC grant GO4-15130A. D.M.A. acknowledges the Science and Technology Facilities Council (STFC) for support through grant ST/L00075X/1. B.L. acknowledges support from the National Natural Science Foundation of China grant 11673010 and the Ministry of Science and Technology of China grant 2016YFA0400702. M.Y.S. and Y.Q.X. acknowledge support from the National Thousand Young Talents program, the 973 Program (2015CB857004), NSFC-11473026, NSFC11421303, the CAS Strategic Priority Research Program (XDB09000000), the Fundamental Research Funds for the Central Universities, and the CAS Frontier Science Key Research Program (QYZDJ-SSW-SLH006). F.E.B. acknowledges support from CONICYT-Chile Basal-CATA PFB-06/ 2007 and FONDECYT Regular 1141218, and the Ministry of Economy, Development, and Tourism's Millennium Science Initiative through grant IC120009, awarded to The Millennium Institute of Astrophysics, MAS. J.-X.W. acknowledges support from NSFC-11233002 and the 973 program (2015CB857005). This project uses Astropy (a Python package; see Astropy Collaboration et al. 2013).

\section{Appendix A Analyses for Star-forming Galaxies}

Here we perform the same analyses as in Section 3 but for star-forming galaxies only, in order to check whether our
Table 3

Numbers of Sources in Different Samples of Star-forming Galaxies

\begin{tabular}{lcccl}
\hline \hline $\begin{array}{l}\text { Sample } \\
(1)\end{array}$ & $\begin{array}{c}\text { Low- } z \\
(2)\end{array}$ & $\begin{array}{c}\text { High- } z \\
(3)\end{array}$ & $\begin{array}{c}\text { Total } \\
(4)\end{array}$ & $\begin{array}{c}\text { Figure } \\
(5)\end{array}$ \\
\hline All & 8149 & 6604 & 14753 & $\mathrm{~N} / \mathrm{A}$ \\
$0.1 M_{\odot} \mathrm{yr}^{-1} \leqslant \mathrm{SFR}<100 M_{\odot} \mathrm{yr}^{-1}$ & 5635 & 6185 & 11820 & 9 \\
$10^{8} M_{\odot} \leqslant M_{*}<10^{11} M_{\odot}$ & 5223 & 5800 & 11023 & 10 and 11 \\
\hline
\end{tabular}

Note. Same format as Table 1, but only for galaxies near the star-forming main sequence.

conclusions also apply for this population alone. We define star-forming galaxies as sources having sSFR within five times (i.e., 0.7 dex) of the median sSFR of the whole sample in the corresponding redshift range. ${ }^{32}$ The median sSFRs for the low$z$ and high $-z$ samples are $10^{-8.94}$ and $10^{-8.72} \mathrm{yr}^{-1}$, respectively. The numbers of star-forming galaxies in the low- and highredshift ranges are 8149 and 6604, respectively (see Table 3).

Similar to Section 3, we bin sources based on SFR $\left(M_{*}\right)$, and split each sample based on $M_{*}$ (SFR). For straightforward comparison with our results for all galaxies, we allow bins with numbers of sources less than 100 in our analyses. Figures 9 and 10 show the results. Similar to the results in Sections 3.1 and 3.2 , sources with higher SFR $\left(M_{*}\right)$ generally have higher $\langle$ BHAR $\rangle$. However, due to the apparent nonlinearity, the linear models in Sections 3.1 and 3.2 result in unacceptable fitting quality, and thus we do not show the fitting results here. From Figure 9 (Figure 10), the nonlinearity mainly arises from the apparent steep rise of $\langle\mathrm{BHAR}\rangle$ above the threshold of $\mathrm{SFR} \sim 10^{0.5} M_{\odot} \mathrm{yr}^{-1}\left(M_{*} \sim 10^{10} M_{\odot} \mathrm{yr}^{-1}\right)$. The steep change might be caused by statistical fluctuations atop a gradual rise, but it could also indicate an intrinsic threshold of SFR $\left(M_{*}\right)$ above which AGN X-ray emission becomes dominant over that of XRBs for star-forming galaxies. Larger samples are needed to differentiate the two possibilities. The SFR- $M_{*}$ relation for star-forming galaxies in Figure 10 (right panels) is more similar to that of B13 compared to that in Figure 5 at the high- $M_{*}$ end (see Section 3.2).

From Figure 9, the high- $M_{*}$ subsamples generally have higher $\langle$ BHAR $\rangle$ than the corresponding low- $M_{*}$ subsamples. From Figure 10, the two subsamples with different SFRs generally have similar $\langle\mathrm{BHAR}\rangle$. These results qualitatively agree with our major conclusion that $\langle\mathrm{BHAR}\rangle$ correlates with $M_{*}$ more strongly than SFR (see Section 3.3). In Figure 10, the low-SFR subsamples of massive galaxies $\left(M_{*} \gtrsim 10^{10} M_{\odot}\right)$ even appear to have higher $\langle\mathrm{BHAR}\rangle$ than their high-SFR counterparts. However, the difference is not statistically significant, and larger samples are needed to clarify this point. From PCOR analyses (see Section 3.3), the parametric method (Pearson) results in $\approx 2-3 \sigma$ significances of the $\langle\mathrm{BHAR}\rangle-M_{*}$ relation in the two redshift ranges in Table 2, while the method shows that the $\langle$ BHAR $\rangle-$ SFR relation is insignificant in both redshift ranges. However, this parametric method models correlations linearly, which is likely not appropriate considering the apparent nonlinearity in Figures 9 and 10. The nonparametric methods

\footnotetext{
32 Different studies often adopt different empirical definitions of star-forming galaxies. Here we adopt a similar definition to that of Elbaz et al. (2011), i.e. the galaxies with sSFR around a typical value. We adopt a wider sSFR range than Elbaz et al. (2011) when defining the main sequence, mostly because our SED-based SFR estimations have larger uncertainties than the FIR-based SFR estimations in Elbaz et al. (2011; see our Section 2.2).
} 

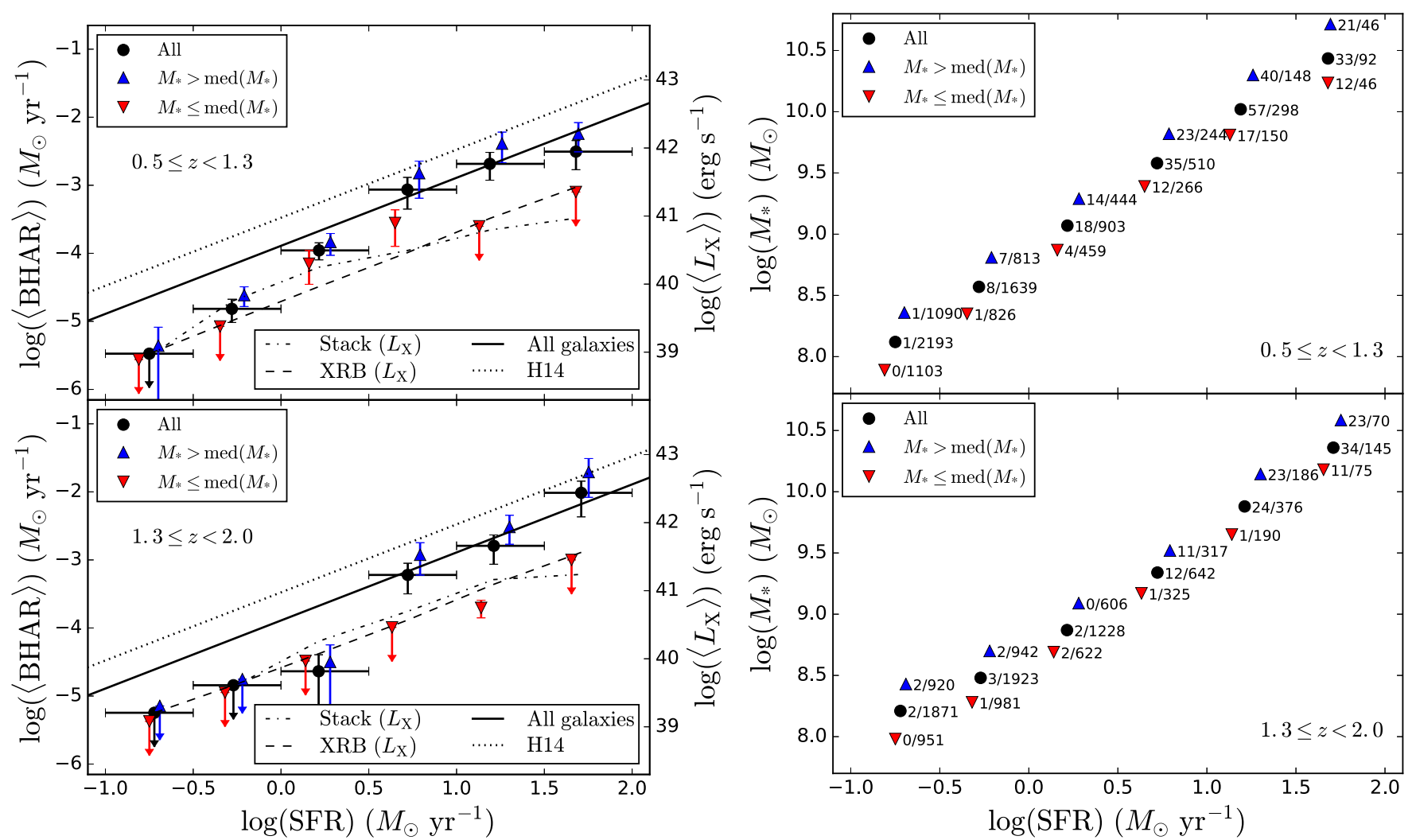

Figure 9. Same format as Figure 4, but for star-forming galaxies. The black solid line indicates the fitting of all galaxies as in Figure 4, rather than the fitting of starforming galaxies.
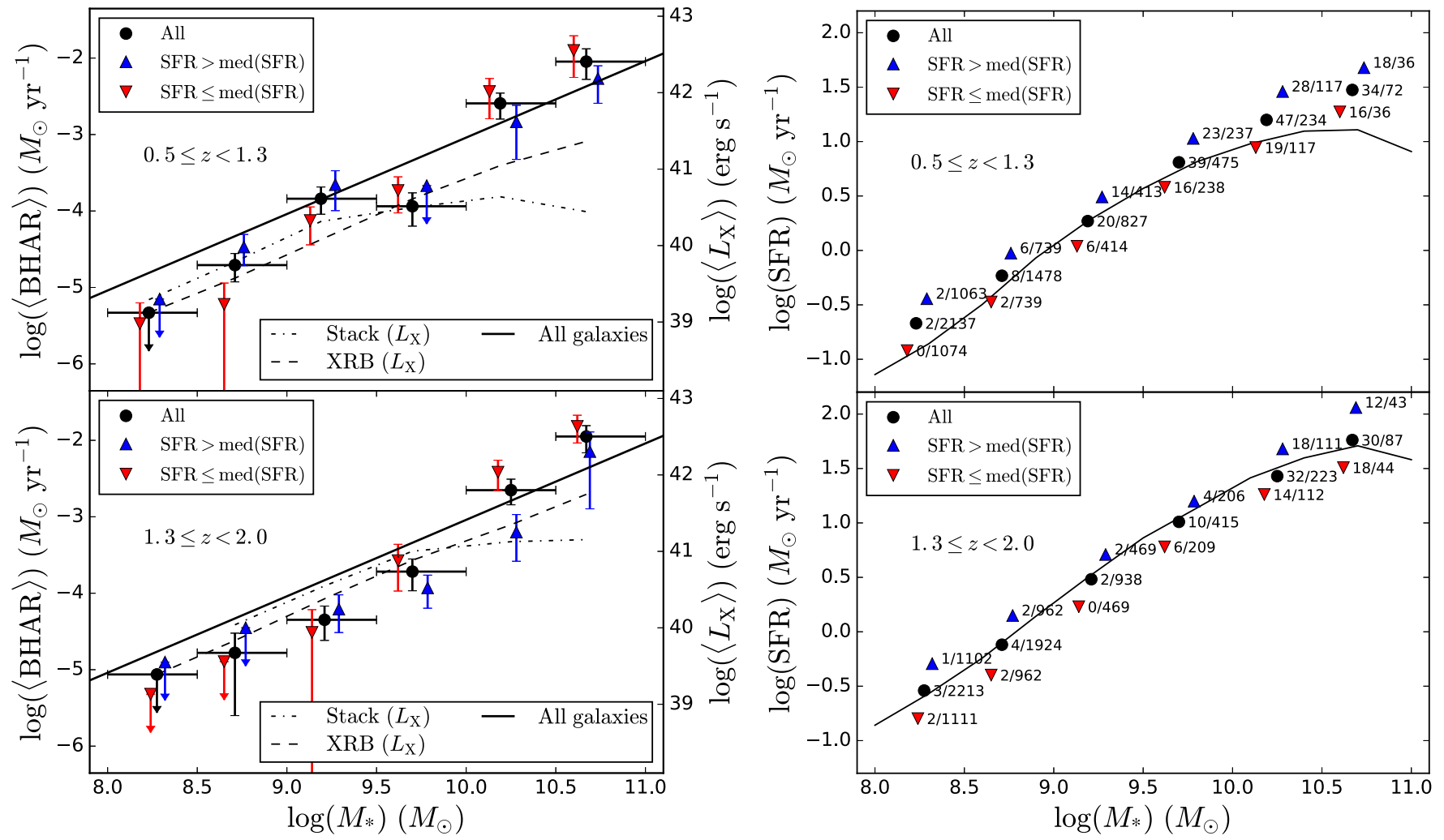

Figure 10. Same format as Figure 5, but for star-forming galaxies. The black solid line indicates the fitting of all galaxies as in Figure 5, rather than the fitting of starforming galaxies. 


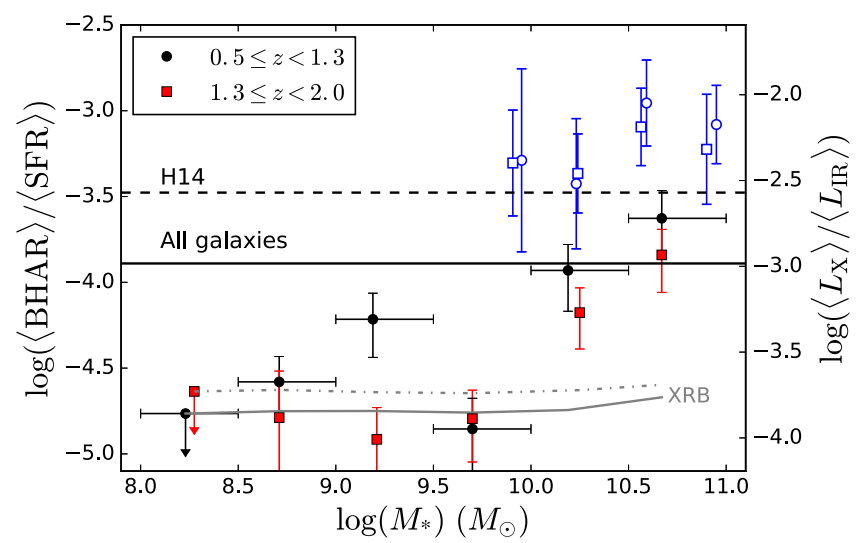

Figure 11. Same format as Figure 8, but for star-forming galaxies. The solid horizontal line indicates the best-fit intercept in Figure 4.

(Spearman and Kendall) do not assume linearity but have less statistical power (see Section 3.3). These methods cannot reveal high significances for either the $\langle\mathrm{BHAR}\rangle-\mathrm{SFR}$ or $\langle\mathrm{BHAR}\rangle-M_{*}$ relations. The reasons are likely to be the reduced sample size (especially the reduced number of AGNs; see Figure 2) and the reduced coverage of the SFR $-M_{*}$ plane.

Figure 11 shows the ratio between $\langle\mathrm{BHAR}\rangle$ and $\langle\mathrm{SFR}\rangle$ for the star-forming galaxies. As in Figure 8 for all galaxies, the ratio is higher for massive galaxies with $M_{*} \gtrsim 10^{10} M_{\odot}$. As expected from Figure 10, there is a sudden "jump" of $\langle\mathrm{BHAR}\rangle /\langle\mathrm{SFR}\rangle$ at $M_{*} \sim 10^{10} M_{\odot}$, probably indicating a physical $M_{*}$ threshold above which AGN activity starts to become very strong. Compared to the corresponding bins in Figure $8,\langle\mathrm{BHAR}\rangle /\langle\mathrm{SFR}\rangle$ values are slightly lower in general, although the trend is weak and within the error bars. This is expected, as the quiescent population has lower $\langle\mathrm{SFR}\rangle$ but similar $\langle$ BHAR $\rangle$ (our main conclusion) compared to the starforming population at given $M_{*}$.

\section{References}

Aird, J., Coil, A. L., \& Georgakakis, A. 2016, MNRAS, 465, 3390 Aird, J., Coil, A. L., Moustakas, J., et al. 2012, ApJ, 746, 90 Aird, J., Nandra, K., Laird, E. S., et al. 2010, MNRAS, 401, 2531 Akylas, A., Georgantopoulos, I., Ranalli, P., et al. 2016, A\&A, 594, A73 Alexander, D. M., Brandt, W. N., Smail, I., et al. 2008, AJ, 135, 1968 Alexander, D. M., \& Hickox, R. C. 2012, NewAR, 56, 93 Alexander, D. M., Stern, D., Del Moro, A., et al. 2013, ApJ, 773, 125 Arnaud, K. A. 1996, in ASP Conf. Ser. 101, Astronomical Data Analysis Software and Systems V, ed. G. H. Jacoby \& J. Barnes (San Francisco, CA: ASP), 17

Astropy Collaboration, Robitaille, T. P., Tollerud, E. J., et al. 2013, A\&A, 558, A33

Azadi, M., Aird, J., Coil, A. L., et al. 2015, ApJ, 806, 187

Bahcall, J. N., Kirhakos, S., Saxe, D. H., \& Schneider, D. P. 1997, ApJ, 479,642

Bañados, E., Venemans, B. P., Decarli, R., et al. 2016, ApJS, 227, 11 Barger, A. J., Cowie, L. L., Owen, F. N., et al. 2015, ApJ, 801, 87 Behroozi, P. S., Wechsler, R. H., \& Conroy, C. 2013, ApJ, 770, 57 (B13) Bellovary, J., Brooks, A., Volonteri, M., et al. 2013, ApJ, 779, 136 Bongiorno, A., Merloni, A., Brusa, M., et al. 2012, MNRAS, 427, 3103 Bonzini, M., Padovani, P., Mainieri, V., et al. 2013, MNRAS, 436, 3759 Borys, C., Smail, I., Chapman, S. C., et al. 2005, ApJ, 635, 853 Brandt, W. N., \& Alexander, D. M. 2015, A\&ARv, 23, 1

Brightman, M., Nandra, K., Salvato, M., et al. 2014, MNRAS, 443, 1999 Bruzual, G., \& Charlot, S. 2003, MNRAS, 344, 1000

Buchner, J., Georgakakis, A., Nandra, K., et al. 2015, ApJ, 802, 89 Calzetti, D., Armus, L., Bohlin, R. C., et al. 2000, ApJ, 533, 682 Calzetti, D., Wu, S.-Y., Hong, S., et al. 2010, ApJ, 714, 1256 Casey, C. M., Narayanan, D., \& Cooray, A. 2014, PhR, 541, 45
Cash, W. 1979, ApJ, 228, 939

Chabrier, G. 2003, ApJL, 586, L133

Chen, C.-T. J., Hickox, R. C., Alberts, S., et al. 2013, ApJ, 773, 3 (C13)

Chiosi, C., \& Carraro, G. 2002, MNRAS, 335, 335

Ciesla, L., Charmandaris, V., Georgakakis, A., et al. 2015, A\&A, 576, A10

Comastri, A., Gilli, R., Marconi, A., Risaliti, G., \& Salvati, M. 2015, A\&A, 574, L10

Croton, D. J., Springel, V., White, S. D. M., et al. 2006, MNRAS, 365, 11

Davis, S. W., \& Laor, A. 2011, ApJ, 728, 98

Delvecchio, I., Lutz, D., Berta, S., et al. 2015, MNRAS, 449, 373

Dubois, Y., Volonteri, M., Silk, J., et al. 2015, MNRAS, 452, 1502

Elbaz, D., Dickinson, M., Hwang, H. S., et al. 2011, A\&A, 533, A119

Fabian, A. C. 2012, ARA\&A, 50, 455

Feigelson, E. D., \& Babu, G. J. 2012, Modern Statistical Methods for Astronomy: With R Applications (Cambridge: Cambridge Univ. Press)

Finoguenov, A., Tanaka, M., Cooper, M., et al. 2015, A\&A, 576, A130

Foreman-Mackey, D., Hogg, D. W., Lang, D., \& Goodman, J. 2013, PASP, 125,306

Fragos, T., Lehmer, B., Tremmel, M., et al. 2013, ApJ, 764, 41

Gebhardt, K., Lauer, T. R., Kormendy, J., et al. 2001, AJ, 122, 2469

Gibson, R. R., Brandt, W. N., \& Schneider, D. P. 2008, ApJ, 685, 773

Gilli, R., Comastri, A., \& Hasinger, G. 2007, A\&A, 463, 79

Greene, J. E., Peng, C. Y., Kim, M., et al. 2010, ApJ, 721, 26

Greene, J. E., Seth, A., Kim, M., et al. 2016, ApJL, 826, L32

Grogin, N. A., Kocevski, D. D., Faber, S. M., et al. 2011, ApJS, 197, 35

Guo, Y., Ferguson, H. C., Giavalisco, M., et al. 2013, ApJS, 207, 24

Häring, N., \& Rix, H.-W. 2004, ApJL, 604, L89

Harris, K., Farrah, D., Schulz, B., et al. 2016, MNRAS, 457, 4179

Harrison, C. M., Alexander, D. M., Mullaney, J. R., et al. 2012, ApJL, 760, L15

Heckman, T. M., \& Best, P. N. 2014, ARA\&A, 52, 589

Hickox, R. C., Mullaney, J. R., Alexander, D. M., et al. 2014, ApJ, 782, 9 (H14)

Ho, L. C. 2007 , ApJ, 669, 821

Hopkins, A. M. 2004, ApJ, 615, 209

Hopkins, P. F., Hernquist, L., Cox, T. J., \& Kereš, D. 2008, ApJS, 175, 356

Hopkins, P. F., Richards, G. T., \& Hernquist, L. 2007, ApJ, 654, 731

Iwasawa, K., Vignali, C., Comastri, A., et al. 2015, A\&A, 574, A144

Jahnke, K., Bongiorno, A., Brusa, M., et al. 2009, ApJL, 706, L215

Johnson, R. A., \& Wichern, D. W. 2002, (Upper Saddle River, NJ: Prentice-Hall)

Jones, M. L., Hickox, R. C., Black, C. S., et al. 2016, ApJ, 826, 12

Juneau, S., Dickinson, M., Bournaud, F., et al. 2013, ApJ, 764, 176

Kauffmann, G., Heckman, T. M., Tremonti, C., et al. 2003, MNRAS, 346, 1055

Kennicutt, R. C., \& Evans, N. J. 2012, ARA\&A, 50, 531

Kennicutt, R. C., Jr. 1998, ApJ, 498, 541

King, A., \& Nixon, C. 2015, MNRAS, 453, L46

Kirkpatrick, A., Pope, A., Alexander, D. M., et al. 2012, ApJ, 759, 139

Kocevski, D. D., Brightman, M., Nandra, K., et al. 2015, ApJ, 814, 104

Koekemoer, A. M., Faber, S. M., Ferguson, H. C., et al. 2011, ApJS, 197, 36 Kormendy, J., \& Ho, L. C. 2013, ARA\&A, 51, 511

Koss, M. J., Assef, R., Baloković, M., et al. 2016, ApJ, 825, 85

Lauer, T. R., Tremaine, S., Richstone, D., \& Faber, S. M. 2007, ApJ, 670, 249

Lehmer, B. D., Basu-Zych, A. R., Mineo, S., et al. 2016, ApJ, 825, 7

Longhetti, M., \& Saracco, P. 2009, MNRAS, 394, 774

Luo, B., Brandt, W. N., Xue, Y. Q., et al. 2010, ApJS, 187, 560

Luo, B., Brandt, W. N., Xue, Y. Q., et al. 2017, ApJS, 228, 2

Lutz, D., Poglitsch, A., Altieri, B., et al. 2011, A\&A, 532, A90

Madau, P., \& Dickinson, M. 2014, ARA\&A, 52, 415

Magdziarz, P., \& Zdziarski, A. A. 1995, MNRAS, 273, 837

Magnelli, B., Popesso, P., Berta, S., et al. 2013, A\&A, 553, A132

Marconi, A., Risaliti, G., Gilli, R., et al. 2004, MNRAS, 351, 169

Martini, P., \& Schneider, D. P. 2003, ApJL, 597, L109

Martis, N. S., Marchesini, D., Brammer, G. B., et al. 2016, ApJL, 827, L25

Marulli, F., Bonoli, S., Branchini, E., Moscardini, L., \& Springel, V. 2008, MNRAS, 385, 1846

Matsuoka, Y., Strauss, M. A., Price, T. N., III, \& DiDonato, M. S. 2014, ApJ, 780,162

Melvin, T., Masters, K., Lintott, C., et al. 2014, MNRAS, 438, 2882

Merloni, A., Bongiorno, A., Bolzonella, M., et al. 2010, ApJ, 708, 137

Merloni, A., Bongiorno, A., Brusa, M., et al. 2014, MNRAS, 437, 3550

Mignoli, M., Cimatti, A., Zamorani, G., et al. 2005, A\&A, 437, 883

Miller, B. P., Gallo, E., Greene, J. E., et al. 2015, ApJ, 799, 98

Mullaney, J. R., Daddi, E., Béthermin, M., et al. 2012a, ApJL, 753, L30

Mullaney, J. R., Pannella, M., Daddi, E., et al. 2012b, MNRAS, 419, 95 
Murphy, K. D., \& Yaqoob, T. 2009, MNRAS, 397, 1549

Mushotzky, R. 2004, in Supermassive Black Holes in the Distant Universe, Vol. 308, ed. A. J. Barger (Dordrecht: Kluwer), 53

Nandra, K., O’Neill, P. M., George, I. M., \& Reeves, J. N. 2007, MNRAS, 382,194

Netzer, H., Lani, C., Nordon, R., et al. 2016, ApJ, 819, 123

Nguyen, D. D., Seth, A. C., den Brok, M., et al. 2017, ApJ, 836, 237

Novak, G. S., Ostriker, J. P., \& Ciotti, L. 2011, ApJ, 737, 26

Pan, Z., Zheng, X. Z., \& Kong, X. 2017, ApJ, 834, 39

Peng, Y., Maiolino, R., \& Cochrane, R. 2015, Natur, 521, 192

Pierce, C. M., Lotz, J. M., Salim, S., et al. 2010, MNRAS, 408, 139

Ranalli, P., Comastri, A., \& Setti, G. 2003, A\&A, 399, 39

Ravikumar, C. D., Puech, M., Flores, H., et al. 2007, A\&A, 465, 1099

Reines, A. E., \& Volonteri, M. 2015, ApJ, 813, 82

Rodighiero, G., Brusa, M., Daddi, E., et al. 2015, ApJL, 800, L10

Rodriguez-Gomez, V., Genel, S., Vogelsberger, M., et al. 2015, MNRAS, 449, 49

Rosario, D. J., Santini, P., Lutz, D., et al. 2012, A\&A, 545, A45

Rosario, D. J., Santini, P., Lutz, D., et al. 2013, ApJ, 771, 63

Saintonge, A., Catinella, B., Cortese, L., et al. 2016, MNRAS, 462, 1749

Santini, P., Ferguson, H. C., Fontana, A., et al. 2015, ApJ, 801, 97

Schawinski, K., Koss, M., Berney, S., \& Sartori, L. F. 2015, MNRAS, 451,2517

Schneider, P. 2014, Extragalactic Astronomy and Cosmology: An Introduction (Berlin: Springer)

Shankar, F., Bernardi, M., Sheth, R. K., et al. 2016, MNRAS, 460, 3119

Shemmer, O., Brandt, W., Paolillo, M., et al. 2014, ApJ, 783, 116

Shen, Y. 2013, BASI, 41, 61

Silverman, J. D., Green, P. J., Barkhouse, W. A., et al. 2008, ApJ, 679, 118

Silverman, J. D., Lamareille, F., Maier, C., et al. 2009, ApJ, 696, 396
Silverman, J. D., Mainieri, V., Salvato, M., et al. 2010, ApJS, 191, 124 Siudek, M., Małek, K., Scodeggio, M., et al. 2016, arXiv:1605.05503

Stanley, F., Harrison, C. M., Alexander, D. M., et al. 2015, MNRAS, 453,591

Stark, A. A., Gammie, C. F., Wilson, R. W., et al. 1992, ApJS, 79, 77

Sun, M., Trump, J. R., Brandt, W. N., et al. 2015, ApJ, 802, 14

Symeonidis, M., Georgakakis, A., Seymour, N., et al. 2011, MNRAS, 417, 2239

Toft, S., Smolčić, V., Magnelli, B., et al. 2014, ApJ, 782, 68

Tomczak, A. R., Quadri, R. F., Tran, K.-V. H., et al. 2014, ApJ, 783, 85

Treister, E., Virani, S., Gawiser, E., et al. 2009, ApJ, 693, 1713

Trump, J. R., Sun, M., Zeimann, G. R., et al. 2015, ApJ, 811, 26

Ueda, Y., Akiyama, M., Hasinger, G., Miyaji, T., \& Watson, M. G. 2014, ApJ, 786, 104

Valluri, M., Ferrarese, L., Merritt, D., \& Joseph, C. L. 2005, ApJ, 628, 137

Vasudevan, R. V., \& Fabian, A. C. 2007, MNRAS, 381, 1235

Vito, F., Gilli, R., Vignali, C., et al. 2016, MNRAS, 463, 348

Vito, F., Maiolino, R., Santini, P., et al. 2014, MNRAS, 441, 1059

Volonteri, M. 2010, A\&ARv, 18, 279

Volonteri, M., Capelo, P. R., Netzer, H., et al. 2015, MNRAS, 452, L6

Volonteri, M., Dubois, Y., Pichon, C., \& Devriendt, J. 2016, MNRAS, 460, 2979

Whitaker, K. E., van Dokkum, P. G., Brammer, G., \& Franx, M. 2012, ApJL, 754, L29

Wu, X.-B., Wang, F., Fan, X., et al. 2015, Natur, 518, 512

Wuyts, S., Förster Schreiber, N. M., Lutz, D., et al. 2011, ApJ, 738, 106

Xu, L., Rieke, G. H., Egami, E., et al. 2015, ApJ, 808, 159

Xue, Y. Q., Brandt, W. N., Luo, B., et al. 2010, ApJ, 720, 368

Yang, G., Brandt, W. N., Luo, B., et al. 2016, ApJ, 831, 145

Yuan, F., \& Narayan, R. 2014, ARA\&A, 52, 529 